\title{
CELL-LIKE EQUIVALENCES AND BOUNDARIES OF CAT(0) GROUPS
}

\author{
CRAIG GUILBAULT AND CHRISTOPHER MOONEY*
}

\begin{abstract}
In 2000, Croke and Kleiner showed that a CAT(0) group $G$ can admit more than one boundary. This contrasted with the situation for $\delta$-hyperbolic groups, where it was well-known that each such group admitted a unique boundary - in a very stong sense. Prior to Croke and Kleiner's discovery, it had been observed by Geoghegan and Bestvina that a weaker sort of uniquness does hold for boundaries of torsion free CAT(0) groups; in particular, any two such boundaries always have the same shape. Hence, the boundary really does carry significant information about the group itself. In an attempt to strengthen the correspondence between group and boundary, Bestvina asked whether boundaries of CAT(0) groups are unique up to cell-like equivalence. For the types of space that arise as boundaries of CAT $(0)$ groups, this is a notion that is weaker than topological equivalence and stronger than shape equivalence.

In this paper we explore the Bestvina Cell-like Equivalence Question. We describe a straightforward strategy with the potential for providing a fully general positive answer. We apply that strategy to a number of test cases and show that it succeeds - often in unexpectedly interesting ways.
\end{abstract}

\section{INTRODUCTION}

One striking difference between the category of negatively curved groups and that of nonpositively curved groups occurs at their ends; whereas a $\delta$-hyperbolic group admits a topologically unique boundary, a $\mathrm{CAT}(0)$ group can admit uncountably many distinct boundaries [5, 25, 15, 17. On its surface, that observation might lead one to believe that a boundary for a CAT(0) group is not a useful object, but that is not the case. Many properties remain constant across the spectrum of boundaries of a given CAT( 0$)$ group, and thus may be viewed as properties of the group itself. One substantial such property, which implies many others, is the shape of the boundary. That observation was made indirectly by Geoghegan $[8$ and, specifically for CAT(0) groups, by Bestvina [1. The upshot is that all boundaries of a given CAT(0) group are topologically similar in a manner made precise by shape theory - a classical branch of geometric topology developed specifically for dealing with spaces with the sort of bad local properties that frequently occur in boundaries of groups. Looking for an even stronger correlation between CAT(0) groups and their boundaries, Bestvina posed the following:

Bestvina's Cell-like Equivalence Question. For a given $C A T(0)$ group $G$, are all boundaries cell-like equivalent?

Precise formulations of the notion of 'shape equivalence' and 'cell-like equivalence' and their relationship to one another will be given shortly. For now we give a quick description of the concept of cell-like equivalence to aid in painting the big picture.

A pair of compacta $X$ and $Y$ are declared to be cell-like equivalent if there exists a third compactum $Z$ and a pair of cell-like maps $X \stackrel{f_{1}}{\longleftarrow} Z \stackrel{f_{2}}{\longrightarrow} Y$. (The reader may temporarily think of a cell-like map as a surjective map with contractible point preimages.) To obtain an equivalence relation we permit several intermediate spaces: $X$ and $Y$ are declared to be cell-like equivalent if there exists a diagram of compacta and cell-like maps of the form:

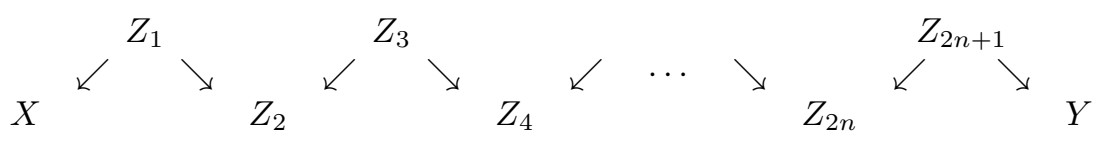

Date: November 2, 2018.

2000 Mathematics Subject Classification. 57M07, 20F65, 54C56.

Key words and phrases. CAT(0) boundary, group boundary, shape equivalence, cell-like equivalence.

*The second author was supported in part by NSF grant EMSW21-RTG: Training the Research Workforce in Geometry, Topology and Dynamics. 
Clearly, cell-like equivalence is weaker than topological equivalence; moreover, if we require that all spaces involved be finite-dimensional, cell-like equivalence is stronger than shape equivalence [21]. In addition to lying between the notions of topological equivalence and shape equivalence, cell-like equivalence has the advantage of allowing an easily understood equivariant variation. Compacta $X$ and $Y$, each equipped with a $G$-action, are declared to be ' $G$-equivariantly cell-like equivalent' if there exists a diagram of type (1D) for which each of the $Z_{i}$ also admits a $G$-action, and each of the cell-like maps respects the corresponding actions. Bestvina has indicated an interest in the following:

Bestvina's Equivariant Cell-like Equivalence Question. For a given CAT(0) group G, are all boundaries G-equivariantly cell-like equivalent?

In this paper we propose a general strategy for obtaining an affimative solution to the equivariant version of Bestvina's question. That strategy is straighforward; it is described at the end of this section. Thus far we are unable to complete the program for arbitrary CAT(0) groups. Instead we present some specific cases where our strategy works - sometimes in surprising ways. In addition we develop some potentially useful generalizations of our approach.

In the remainder of this introduction we review some basic notions related to shape theory and cell-like equivalences. We then discuss CAT(0) spaces and groups enough so we can go on to describe our standard strategy for obtaining equivariant cell-like equivalences between pairs of boundaries. Lastly we outline the main examples and results to be presented in the remainder of the paper.

By compactum we mean a compact metric space. There are a variety of ways of saying what it means for compacta $X$ and $Y$ to be shape equivalent (denoted $X \stackrel{\text { sh }}{\sim} Y$ ). One method, due to Chapman [4], involves the Hilbert cube $Q=\prod_{i=1}^{\infty}[0,1]$. Embed $X$ and $Y$ in as $\mathcal{Z}$-sets (for example, place $X$ and $Y$ in $\left.\{0\} \times \prod_{i=2}^{\infty}[0,1] \subseteq Q\right)$. Then $X \stackrel{\text { sh }}{\sim} Y$ if and only if $Q-X$ is homeomorphic to $Q-Y$. If $X$ and $Y$ are both finite-dimensional one can avoid infinite dimensional topology by embedding $X$ and $Y$ nicely in $\mathbb{R}^{n}$, where $n$ is large compared to the dimensions of $X$ and $Y$. Then $X \stackrel{\text { sh }}{\sim} Y$ if and only if $\mathbb{R}^{n}-X$ is homeomorphic to $\mathbb{R}^{n}-Y$. See 21] for details.

Another way to characterize shape is more complex, but often easier to apply. Given a compactum $X$, we first choose an associated inverse sequence of finite polyhedra and continuous maps

$$
K_{0} \stackrel{f_{1}}{\longleftarrow} K_{1} \stackrel{f_{2}}{\longleftarrow} K_{2} \stackrel{f_{2}}{\longleftarrow} \cdots .
$$

If $X$ happens to arise as an inverse limit of finite polyhedra, then that sequence may be chosen as the associated inverse sequence. Another way to obtain an associated inverse sequence for $X$ is to choose a sequence of finite covers $\left\{\mathcal{U}_{i}\right\}_{i=0}^{\infty}$ of $X$ by $\varepsilon_{i}$-balls such that $\varepsilon_{i} \rightarrow 0$ and each $\mathcal{U}_{i}$ refines $\mathcal{U}_{i-1}$; then, for each $i$, let $K_{i}$ be the nerve of $\mathcal{U}_{i}$ and $f_{i}$ be the corresponding simplicial map. Yet another way of obtaining an associated inverse sequence can be applied when $X$ is finite-dimensional: embed $X$ in $\mathbb{R}^{n}$ and let $\left\{K_{i}\right\}_{i=0}^{\infty}$ be a decreasing sequence of polyhedral neighborhoods of $X$ with all bonding maps being inclusions.

Given an increasing sequence $\left\{i_{k}\right\}_{k=0}^{\infty}$ of natural numbers, there is a corresponding subsequence of (2)

$$
K_{i_{0}} \stackrel{f_{i_{1}, i_{0}}}{\longleftarrow} K_{i_{1}} \stackrel{f_{i_{2}, i_{1}}}{\longleftarrow} K_{i_{2}} \stackrel{f_{i_{3}, i_{2}}}{\longleftarrow} \cdots
$$

where, for any integers $n>m, f_{n, m}$ is the obvious composition of the $f_{j}$ taking $K_{n}$ to $K_{m}$. Declare a pair of inverse sequences $\left\{K_{i}, f_{i}\right\}$ and $\left\{L_{i}, g_{i}\right\}$ to be pro-equivalent if they contain subsequences that fit into a diagram of the form

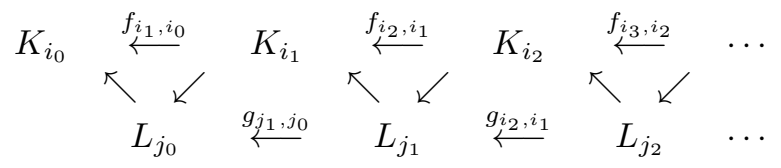

where each triangle is only required to commute up to homotopy. Now $X \stackrel{\text { sh }}{\sim} Y$ if and only if associated inverse sequences are pro-equivalent.

Example 1.1. By using the inverse sequence approach, it is easy to see that the following examples each has the shape of a circle. 


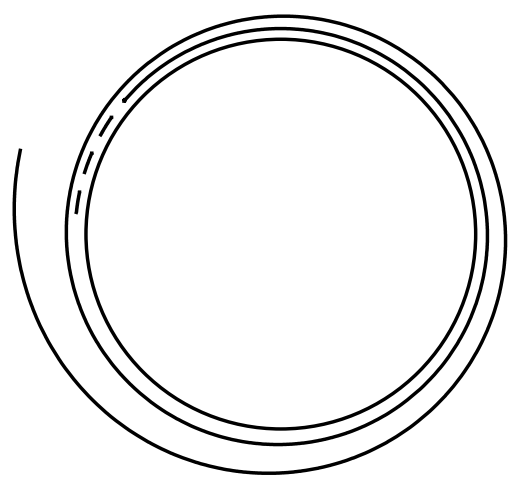

Figure 1. Ferry Spiral

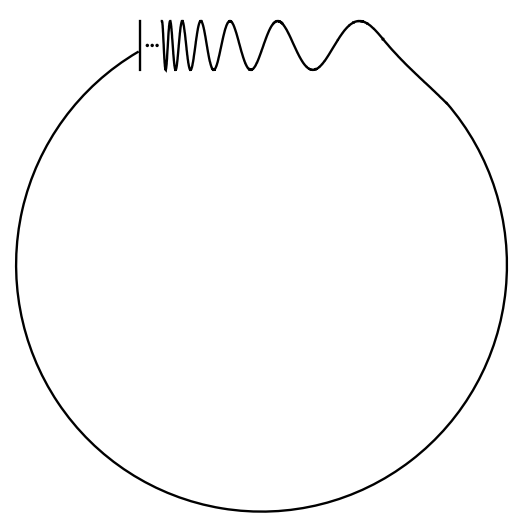

Figure 2. Warsaw Circle

Example 1.2. By repeated application of Borsuk's homotopy extension property, one sees that every contractible compactum has the same shape as a point; these are the prototypical comapcta with trivial shape. An example of a non-contractible compactum with the shape of a point is the topologist's sine curve:

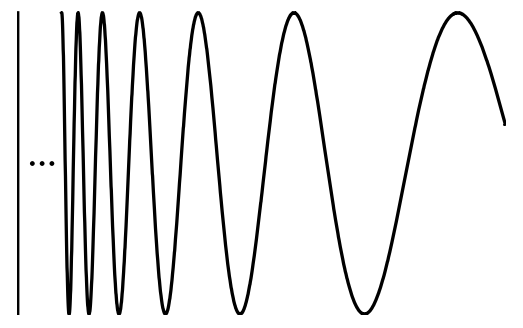

Figure 3. Topologist's Sine Curve

1.1. The notion of cell-like equivalence. A compactum is cell-like if it has the shape of a point. A map $f: X \rightarrow Y$ between compacta is cell-like if $f^{-1}(y)$ is cell-like for each $y \in Y$. Cell-like maps have been studied extensively - they play a central role in manifold topology. Compacta $X$ and $Y$ are cell-like equivalent if there exists a finite sequence of compacta $Z_{1}, \cdots, Z_{2 n+1}(n \geq 0)$ and cell-like maps as described by diagram (1); in this case we write $X \stackrel{\mathrm{CE}}{\sim} Y$. When $X$ and $Y$ are finite-dimensional, the existence of a cell-like map $f: X \rightarrow Y$ implies $X \stackrel{\text { sh }}{\sim} Y$; thus, if $X$ and $Y$ are 'cell-like equivalent through finite-dimensional compacta' (there exists a diagram of type (11) for which all spaces are finite-dimensional), then $X$ and $Y$ have the same shape. Since all boundaries of CAT(0) groups are finite-dimensional [24, as are all intermediate spaces utilized in this paper, we generally think of 'cell-like equivalence' as being stronger than 'shape equivalence'.

A famous counterintuitive example helps to illustrate the above definition.

Example 1.3. Let $A=[0,1] \times\{0\} \subseteq \mathbb{R}^{2}$ and $C \subseteq A$ be the middle-thirds Cantor set; let $B \subseteq \mathbb{R}^{2}$ be the cone over $C$ with cone-point $\left(\frac{1}{2}, 1\right)$. Let $Z=A \cup B$. Since $A$ and $B$ are contractible the quotient maps $Z \rightarrow Z / A$ and $Z \rightarrow Z / B$ are cell-like. The image of the latter is the standard Hawaiian earring with countably many loops; call this space $X$. Notice that $Z / A$ is homeomorphic to $\Sigma C$, the suspension of a Cantor set. By crushing out a single suspension arc, we get a cell-like map $\Sigma C \rightarrow Y$ where $Y$ is a 'bigger Hawaiian earring', having a Cantor set's worth of loops. Putting all of this together, we get that $X$ and $Y$ are cell-like and shape equivalent (see Figure 4).

Example 1.4. An easy application of Example 1.2 shows that the Warsaw circle (see Figure 2) is cell-like equivalent to an ordinary circle. In 7], it is shown that the Ferry Spiral (see Figure 1) is not cell-like equivalent to a circle. 

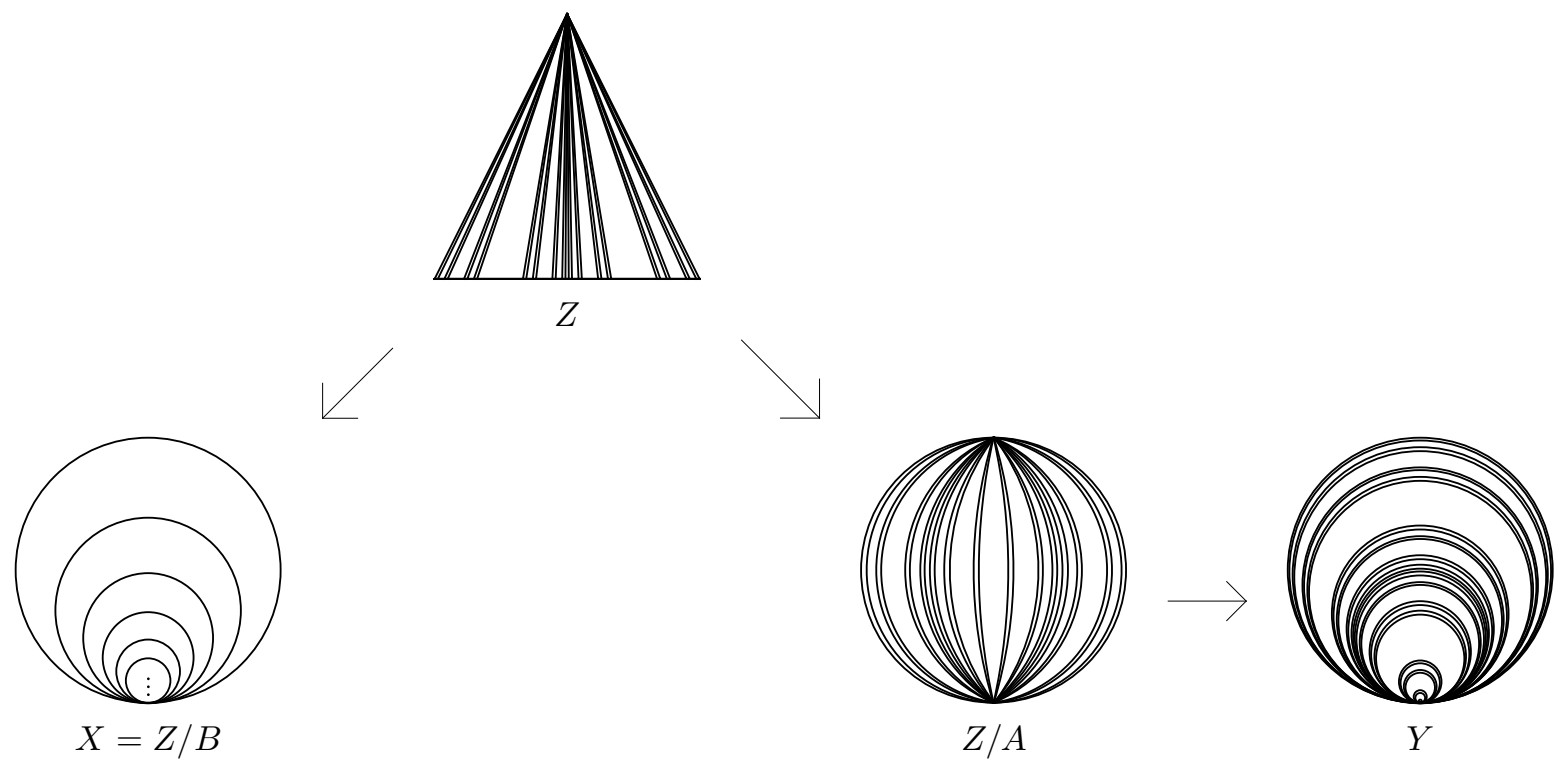

Figure 4. CE Hawaiian Earrings

As noted in the introduction, the notion of cell-like equivalence lends itself nicely to an equivariant version. Suppose compacta $X$ and $Y$ each admit a $G$-action and suppose there exists a diagram of the form (1) where each of the compacta $Z_{i}$ also admits a $G$-action and each of the maps in that diagram commutes with the appropriate actions. We say that $X$ and $Y$ are $G$-equivariantly cell-like equivalent and we write $X \stackrel{G \text {-CE }}{\sim} Y$.

1.2. CAT(0) groups and their boundaries. A geodesic metric space $X$ is called a $C A T(0)$ space if each of its triangles is at least as thin as the corresponding comparison triangle in the Euclidean plane. A group $G$ is called a $C A T(0)$ group if it acts geometrically (properly and cocompactly via isometries) on a proper CAT(0) space. Such a space $X$ can be compactified by the addition of its visual boundary $\partial X$ which may be defined as the space of all equivalence classes of geodesic rays in $X$, where a pair of rays $\alpha, \beta:[0, \infty) \rightarrow X$ are equivalent if they are asymptotic, i.e., if $\{d(\alpha(t), \beta(t) \mid t \in[0, \infty))\}$ is bounded above. When $G$ acts geometrically on $X$ we call $\partial X$ a boundary for $G$. Clearly, the action of $G$ on $X$ induces an action by $G$ on $\partial X$. An alternative, but equivalent, approach to defining the visual boundary declares $\partial X$ to be the collection of all geodesic rays emanating from a fixed base point $x_{0} \in X$. This simplifies matters since no equivalence relation is needed; we will use the latter approach when convenient. We put the cone topology on $\partial X$ which roughly says that two geodesic rays are close in $\partial X$ if they track together for a long time before they diverge. More details on CAT(0) spaces and their boundaries, including a discussion of the topology on $\bar{X}=X \cup \partial X$, will be presented as necessary. In addition, the reader may wish to consult 3 .

Nonuniqueness of the boundary of a $\operatorname{CAT}(0)$ group $G$ is possible since $G$ can act on more than one $\operatorname{CAT}(0)$ space. When the action by $G$ is free, covering space techniques and other topological tools allowed Bestvina [1] to show that all boundaries of $G$ are shape equivalent. Later, Ontaneda [18] extended that obsevation to include all $\mathrm{CAT}(0)$ groups. In those cases where all $\mathrm{CAT}(0)$ boundaries of a given $G$ are homeomorphic we say that $G$ is rigid. Clearly Bestvina's Cell-like Equivalence Question has a positive answer for all such groups. A positive answer has also been given for groups which split as products with infinite factors [16].

When a group $G$ acts nicely on multiple spaces, a key relationship between those spaces is captured by the notion of 'quasi-isometry'. A function $f: X \rightarrow X^{\prime}$ between metric spaces is called a quasi-isometric embedding (QIE) if there exist positive constants $\lambda$ and $\varepsilon$ such that for all $x, y \in X$

$$
\frac{1}{\lambda} d(x, y)-\varepsilon \leq d^{\prime}(f(x), f(y)) \leq \lambda d(x, y)+\varepsilon .
$$

If, in addition, there exists a $C>0$ such that for every $z \in X^{\prime}$, there is some $x \in X$ such that $d^{\prime}(f(x), z) \leq C$, then we call $f$ a quasi-isometry and declare $X$ and $X^{\prime}$ to be quasi-isometric. 
By choosing a finite generating set and endowing it with the corresponding word metric, any finitely generated group can be viewed as a metric space. It follows from the Švarc-Milnor Lemma that, up to quasi-isometry, this metric space is independent of the choice of generating set; in fact if $X$ is any length space on which $G$ acts geometrically, then for any base point $x_{0} \in X$ the orbit map $G \rightarrow X$ given by $g \mapsto g x_{0}$ is a quasi-isometry [23, 14].

Given a subset $A$ of a CAT(0) space $X$, define the limset of $A$ to be the collection of all limit points of $A$ lying in $\partial X$, in other words, limset $A=\bar{A}-X$ where the closure is taken in $\bar{X}$. Clearly any such limset is a closed subset of $\partial X$. If $G$ acts on a proper $\operatorname{CAT}(0)$ space properly discontinuously by isometries, then we denote by $\operatorname{limset}(X, G)$ the limset of the image of $G$ under the orbit map. This provides a compactification $G \cup \operatorname{limset}(X, G)$ for $G$. Note that if this action is geometric then $\operatorname{limset}(X, G)=\partial X$. If $G$ acts properly discontinuously on two proper $\operatorname{CAT}(0)$ spaces $X$ and $Y$, then it is natural to compare the two compactifications $\partial X$ and $\partial Y$. If the identity map on $G$ extends continuously to a map $G \cup \partial X \rightarrow G \cup \partial Y$, then the restriction $\partial X \rightarrow \partial Y$ is called a limset map. The existence of such a map is very strong. It means that whenever an unbounded sequence of group elements converges in one compactification, it also converges in the other (see Lemma 3.2). Two limsets are considered equivalent if there is a limset map between them which is a homeomorphism.

We call $G$ strongly rigid if whenever $G$ acts geometrically on proper $\operatorname{CAT}(0)$ spaces $X$ and $Y$, the boundaries $\partial X$ an $\partial Y$ are equivalent in the above sense. Examples of such groups include free abelian groups, $\delta$-hyperbolic CAT(0) groups (hereafter referred to as negatively curved groups), and others [12, 11]. Clearly Bestvina's Equivariant Cell-like Equvalence Question has a positive answer for all strongly rigid groups.

If $G$ acts properly discontinuously by isometries on $X$, then so does any subgroup $H \leq G$. If $H$ has infinite index, then it does not act geometrically, since cocompactness has been lost. Moreover, even when $H$ is finitely generated, it is not always the case that $H \hookrightarrow G$ (or equivalently $h \mapsto h x_{0}$ ) is a QIE. An object of special interest to us will be limset $H$ for certain subgroups $H$ of CAT(0) groups.

Beyond the above mentioned examples of strongly rigid groups, the question of when two boundaries of a CAT(0) group are equivalent has been studied by Croke and Kleiner for a class of groups including graph manifold groups [6], and by Hosaka in more generality [10].

1.3. The standard strategy and our Main Conjecture. Suppose $G$ acts geometrically on a pair of proper CAT(0) spaces $X_{1}$ and $X_{2}$. Then the $l_{2}$-metric $d=\sqrt{d_{1}^{2}+d_{2}^{2}}$ makes $X_{1} \times X_{2}$ a proper CAT(0) space on which $G \times G$ acts geometrically via the product action. It is a standard fact that $\partial\left(X_{1} \times X_{2}\right)$ is homeomorphic to the topological join of the original boundaries [3, Example II.8.11(6)]. To see this, first choose a base point $\left(x_{1}, x_{2}\right) \in X_{1} \times X_{2}$ and define slopes of segments and rays in $X_{1} \times X_{2}$ based at $\left(x_{1}, x_{2}\right)$ in the obvious way. A ray $\alpha$ may be projected into $X_{1}$ and $X_{2}$ to obtain a pair of rays $\alpha_{1}$ and $\alpha_{2}$-except in those cases where the slope is 0 or $\infty$ which produce an $\alpha_{i}$ that is constant. Assign to each $\alpha$ three coordinates: $\alpha_{1}, \alpha_{2}$, and the slope of $\alpha$. Keeping in mind the exceptional cases where $\alpha$ has slope 0 or $\infty$, we get a correspondence between $\partial\left(X_{1} \times X_{2}\right)$ and the quotient space

$$
\partial X_{1} * \partial X_{2}=\partial X_{1} \times \partial X_{2} \times[0, \infty] / \sim
$$

where $\left(\alpha_{1}, \alpha_{2}, 0\right) \sim\left(\alpha_{1}, \alpha_{2}^{\prime}, 0\right)$ for all $\alpha_{2}, \alpha_{2}^{\prime} \in \partial X_{2}$ and $\left(\alpha_{1}, \alpha_{2}, \infty\right) \sim\left(\alpha_{1}^{\prime}, \alpha_{2}, \infty\right)$ for all $\alpha_{1}, \alpha_{1}^{\prime} \in \partial X_{2}$. This join contains a preferred copy of $\partial X_{1}$ (all points with slope 0 ) and a preferred copy of $\partial X_{2}$ (all points with slope $\infty$ ) which may be identified with the boundaries of convex subspaces $X_{1} \times\left\{x_{2}\right\}$ and $\left\{x_{1}\right\} \times X_{2}$. A more thorough development of the the notion of slope may be found in Section 4 .

Now consider the diagonal subgroup $G^{\Delta}=\{(g, g) \mid g \in G\}$ of $G \times G$. Clearly, $G^{\Delta}$ is isomorphic to $G$ and acts on $X_{1} \times X_{2}$ properly by isometries. For $g \in G$, we will denote $g^{\Delta}=(g, g)$. In Section 4.1] we make the following observations:

(i) The map $g \longmapsto g^{\Delta}\left(x_{1}, x_{2}\right)$ is a QIE of $G$ in $X_{1} \times X_{2}$, and

(ii) limset $G^{\Delta}$ is a closed subset of $\partial X_{1} * \partial X_{2}$ that misses the preferred copies of $\partial X_{1}$ and $\partial X_{2}$.

We refer to limset $G^{\Delta}$ as a schmear of $\partial X_{1}$ and $\partial X_{2}$. Item (i) is used in proving (ii) and offers hope that $\Lambda$ resembles a boundary for $G$. Item (ii) allows us to restrict the projections of $\partial X_{1} \times \partial X_{2} \times(0, \infty)$ onto $\partial X_{1}$ and $\partial X_{2}$ to obtain a pair of $G$-equivariant schmear maps $\phi_{1}: \Lambda \rightarrow \partial X_{1}$ and $\phi_{2}: \Lambda \rightarrow \partial X_{2}$.

Our standard strategy is summed up by the following: 
Main Conjecture. Suppose $G$ acts geometrically on a pair of $C A T(0)$ spaces $X_{1}$ and $X_{2}$. Then both schmear maps are cell-like; hence $\partial X_{1}$ and $\partial X_{2}$ are G-equivariantly cell-like equivalent.

1.4. The main results. As noted earlier, we are not yet able to make the above program work in full generality. In this paper, we provide positive evidence for our approach by presenting an array of interesting examples and proving the conjecture for a specific class of groups.

One simple, but revealing, example involves the negatively curved group $\mathbb{F}_{2}$ which has boundary homeomorphic to a Cantor set $C$. By strong rigidity for $\delta$-hyperbolic groups even the Equivariant Bestvina Question is not in doubt here; one might even expect any schmear for $\mathbb{F}_{2}$ to be just another copy of $C$. On the contrary, by choosing different $\operatorname{CAT}(0)$ spaces on which $\mathbb{F}_{2}$ acts, the resulting schmear is often not a Cantor set, but rather, is homeomorphic to $C \times[0,1]$. In those cases, all point preimages are copies of $[0,1]$. See Example 2.4 Indeed we can compute schmears of CAT(0) boundaries of negatively curved groups by applying recent work of Link [13] (see Corollary 4).

In a rather different way the group $\mathbb{F}_{2} \times \mathbb{Z}$ admits an interesting schmear. Here we can construct a nontrivial schmear for a pair of boundaries where the two boundaries are the boundary of the same space, showing that the schmear depends not only on the underlying spaces themselves, but also on the action chosen. In [2] Bowers and Ruane utilized a standard and a twisted action of $\mathbb{F}_{2} \times \mathbb{Z}$ on $T \times \mathbb{R}$, where $T$ is the standard valence four tree with edge lengths equal to 1 , to show that this group is rigid but not strongly rigid. Inserting those actions into our program produces a pair of schmear maps for which some point preimages are intervals and the rest are singletons. In some sense the schmear and its corresponding maps provide a missing link between the standard and twisted action. See Section 2.2 .

Our first theorem proves the existence of schmears. Note that the statement applies to a broader class of group actions on $\mathrm{CAT}(0)$ spaces than simply geometric actions.

Theorem 1. Assume an infinite group $G$ acts on $C A T(0)$ spaces $X_{1}$ and $X_{2}$ such that $G \rightarrow X_{1}$ and $G \rightarrow X_{2}$ are QIEs. Then there exists an action of $G$ by isometries on a third $C A T(0)$ space $X$ such that $G \rightarrow X$ is a QIE and there are natural limset maps limset $G \rightarrow \partial X_{i}$. If the action of $G$ on both $X_{i}$ is by semi-simple isometries, then so is the action on $X$.

It follows from [2] that Bestvina's Equivariant Cell-Like Equivalence Question has a positive answer for products of negatively curved groups with free abelian groups. They prove that any pair of boundaries is equivariantly homeomorphic. Since this equivariant homeomorphism does not come from the orbit map, this next theorem, which verifies the Main Conjecture for a particular subclass of such groups, is stronger.

Theorem 2. Assume $G=\mathbb{F}_{m} \times \mathbb{Z}^{d}$ acts geometrically on two $C A T(0)$ spaces $X_{1}$ and $X_{2}$ and $\Lambda$ be the schmear of the pair $\partial X_{1}, \partial X_{2}$. Then point preimages for the schmear maps $\Lambda \rightarrow \partial X_{i}$ are topological cells.

In other words, regardless of the CAT(0) spaces and geometric actions chosen, point preimages under the schmear maps are homeomorphic to cells of various dimensions. This is somewhat surprising when compared to recent work by Staley [22] who uses these same groups to realize some exotic limsets for images of geodesic rays under equivariant quasi-isometries. The lesson learned from our theorem seems to be that "taking the whole schmear" has a tendency to paint over oddities in the local behavior of limsets. Close examination of the examples and results presented here will help make sense of this last comment.

Our final result addresses a natural question to this approach. We have constructed a single intermediate compactum $Z$ admitting an action by the group $G$ in question by letting $Z$ be the limset of $G$ under some non-cocompact action. One may ask "Can $Z$ be realized as a boundary of G?" The answer is "No". If we seek to find equivariant cell-like equivalences between two boundaries of $G$ by taking the extensions of QIEs, then we must pass through limsets of non-cocompact actions which cannot themselves be realized as boundaries of the group in question.

Theorem 3. Let $\mathbb{F}_{2} \times \mathbb{Z}$ act geometrically on two $C A T(0)$ spaces $X$ and $Y$. If there is a limset map $\partial X \rightarrow \partial Y$, then that limset map is a homeomorphism.

Remark 1.5. If $H \leq G$ is a finite index subgroup of a group $G$, then information about $G$ gives information about $H$ - if $G$ is $\operatorname{CAT}(0)$, then so is $H$ and every boundary of $G$ is also a boundary of $H$. The reverse direction is not understood. It is currently an open question whether $H$ being $\operatorname{CAT}(0)$ implies that $G$ is also 
CAT(0), and there are cases where $H$ and $G$ are both CAT(0), but $G$ has fewer boundaries than $H$. One nice aspect of the approach taken in this paper is that all of our results which apply to a given CAT(0) group $\mathrm{H}$ are immediately valid for any $\mathrm{CAT}(0)$ group containing $\mathrm{H}$ as a finite index subgroup (see Proposition 3.12).

Acknowledgements. We thank Kim Ruane.

\section{EXAMPLES}

In this section we provide concrete examples of the interesting end behavior of some simple CAT $(0)$ groups as well as schmears.

2.1. Boundaries of $\mathbb{F}_{2} \times \mathbb{Z}$. It was first observed by Bowers and Ruane in 2] that in contrast to the situation for negatively curved and free abelian groups, equivariant quasi-isometries need not extend to homeomorphisms of boundaries. This is true even for $\mathbb{F}_{2} \times \mathbb{Z}$, which is perhaps the simplest example of a $\operatorname{CAT}(0)$ group which is neither negatively curved nor free abelian. In this section we provide several variations on this example.

The main theorem of [2] says that whenever $\mathbb{F}_{2} \times \mathbb{Z}$ acts geometrically on a $\operatorname{CAT}(0)$ space $X, \partial X$ is homeomorphic to the suspension of the Cantor set. By the Flat Torus Theorem, one can assume that $X$ splits as a product $Y \times \mathbb{R}$ where $\mathbb{Z}$ is generated by a translation in the $\mathbb{R}$-coordinate and the action of $\mathbb{F}_{2}$ projects to a geometric action on $Y$. The suspension points of $\partial X$ are called poles. The subspace $\partial Y$, which is homeomorphic to the Cantor set, is called the equator. The suspension arcs are called longitudes.

Typically longitudes are parameterized using angles. For us it will be convenient to parameterize them in terms of slopes:

$$
\partial X=\partial Y \times[-\infty, \infty] / \sim
$$

where $\sim$ collapses the sets $Y \times\{\infty\}$ and $Y \times\{-\infty\}$ to the poles. Take a geodesic ray $\alpha$ based at a point $\left(y_{0}, 0\right) \in Y \times \mathbb{R}$ which does not go to a pole. Let $\alpha_{Y}$ be its projection onto $Y \times\{0\} ; \alpha_{Y}(\infty)$ is a point of the equator. Then $\alpha$ lives in the half-plane $F=\alpha_{Y} \times \mathbb{R}$ and has a slope $M(\alpha)$ defined in terms of these coordinates. The boundary of $F$ is the longitude containing $\alpha(\infty)$. Given $\zeta \in \partial X$, we will denote by $l(\zeta)$ the longitude containing $\zeta$ and by $l(\zeta, M)$ the point of $l(\zeta)$ corresponding to a ray of slope $M$. So $l(\zeta, \pm \infty)$ are the poles and $l(\zeta, 0)$ lies in the equator.

Example 2.1 (Twisting). Let $a$ and $b$ generate $\mathbb{F}_{2}$ and $c$ denote the generator of $\mathbb{Z}$. Let $\Gamma$ denote the Cayley graph of $\mathbb{F}_{2}$, an infinite 4-valent tree with $v \in \Gamma$ a vertex. Then we have a natural product action of $\mathbb{F}_{2} \times \mathbb{Z}$ on $X=\Gamma \times \mathbb{R}$ by letting $c$ translate $\mathbb{R}$ one unit. We will denote the action of an element $g$ on a point $x \in X$ by $g \cdot x$. In coordinates, we have

$$
\begin{aligned}
a \cdot(v, t) & =(a v, t), \\
b \cdot(v, t) & =(b v, t), \\
\text { and } c \cdot(v, t) & =(v, t+1) .
\end{aligned}
$$

Now we consider another action on the same space by "twisting" one of the generators. Specifically, we change the action by letting the action of $b$ on the $\mathbb{R}$-coordinate be the same translation as $c$. We denote the resulting action by $*$. So we now have

$$
\begin{aligned}
a *(v, t) & =(a v, t), \\
b *(v, t) & =(b v, t+1), \\
\text { and } c *(v, t) & =(v, t+1) .
\end{aligned}
$$

Now if the identity map $G \rightarrow G$ is to extend to a continuous function between these two boundaries, it must be the case that if a sequnce of group elements converges to a point of $\partial X$ in the first action, then it also converges to a point of $\partial X$ in the second action. We claim that this is not the case.

Our first observation is that in $\Gamma$, the sequences $\left(a^{n} v\right)$ and $\left(a^{n} b^{n^{2}} v\right)$ converge to the same point of $\partial \Gamma$; we will call this point $a^{\infty}$. In $X$ it follows that the sequences $\left(a^{n} \cdot x\right)$ and $\left(a^{n} b^{n^{2}} \cdot x\right)$ have their limit point in $l\left(a^{\infty}\right)$. In fact they converge to the same point - since they both only act in the $\Gamma$-coordinate, they both converge to $l\left(a^{\infty}, 0\right)$. 
Now consider the second action $G * X$. The sequence $\left(a^{n} * x_{0}\right)$ also converges to $l\left(a^{\infty}, 0\right)$. But this time $\left(a^{n} b^{n^{2}} * x_{0}\right)$ converges to a ray of slope 1 . For, the slope of the line segment $\left[x_{0}, a^{n} b^{n^{2}} * x_{0}\right]$ is

$$
\frac{n^{2}}{n+n^{2}} \rightarrow 1
$$

This means that the sequences $\left(a^{n}\right)$ and $\left(a^{n} b^{n^{2}}\right)$ converge to the same boundary point in one action and two different points in the other action. So there is no limset map $\partial X \rightarrow \partial X$ which respects the group actions.

Given $\zeta \in \partial X$, let $\Lambda(\zeta)$ denote the subset of $\partial X$ consisting of points $\zeta^{\prime}$ for which there is a sequence $\left(g_{n}\right)$ such that $g_{n} \cdot x \rightarrow \zeta$ and $g_{n} * x \rightarrow \zeta^{\prime}$. Then $\Lambda\left(a^{\infty}\right)$ is an interval in the longitude of $a^{\infty}$ stretching between $l\left(a^{\infty}, 1\right)$ and $l\left(a^{\infty},-1\right)$. The sequences in $\mathbb{F}_{2}$ converging to $a^{\infty}$ in the action $G \cdot X$ are those of the form $a^{k_{n}} w_{n}$ where $k_{n} \rightarrow \infty$. But under the action $G * X$, such sequences have varying slope depending on the asymptotic ratio of $b$ 's to $a$ 's.

Finally we note that the image of the map $\mathbb{F}_{2} \rightarrow X$ under the twisted action is not quasi-convex, even though it is a QIE. To see that the $\mathbb{F}_{2}$-orbit of a point is not quasi-convex under $*$, observe that the geodesic $\left[b^{n} * x_{0}, a b^{n} * x_{0}\right]$ strays farther and farther from $x_{0}$ as $n$ gets large. But it is a QIE because

$$
l(w) \leq d_{X}\left(x_{0}, w * x_{0}\right) \leq \sqrt{2} l(w)
$$

where $w \in \mathbb{F}_{2}$ and $l$ denotes wordlength in $\mathbb{F}_{2}$ with respect to the generating set $\{a, b\}$. This stands in contrast to the situation for $\delta$-hyperbolic spaces, where the concepts of quasi-convexity and QIE are equiavlent [3, Corollary III.Г.3.6].

Example 2.2 (Stretching). Here is a different type of phenomenon. Let $\Gamma^{\prime}$ be a homeomorphic copy of $\Gamma$ in which the edges corresponding to $a$ have length 1 (as before) and edges corresponding to $b$ have length 2 . Let $X^{\prime}=\Gamma^{\prime} \times \mathbb{R}$ and $G$ act on $X^{\prime}$ via the product action. We will denote this action by $\odot$. Let $x_{0}^{\prime} \in X^{\prime}$ be the preferred basepoint $\left(y_{0}^{\prime}, 0\right)$ where $y_{0}^{\prime} \in \Gamma^{\prime}$ is the vertex corresponding to $y_{0}$. Since $\mathbb{F}_{2}$ acts only in the $\Gamma^{\prime}$-coordinate, the quasi-isometry $\mathbb{F}_{2} \cdot x_{0} \rightarrow \mathbb{F}_{2} \odot x_{0}^{\prime}$ extends to a map between the equators. However the quasi-isometry

$$
\left(\mathbb{F}_{2} \times \mathbb{Z}\right) \cdot x_{0} \rightarrow\left(\mathbb{F}_{2} \times \mathbb{Z}\right) \odot x_{0}^{\prime}
$$

does not extend. In $X$, the sequences $\left(a^{n} c^{n} \cdot x_{0}\right)$ and $\left(a^{n} b^{n^{2}} c^{n^{2}} \cdot x_{0}\right)$ both converge to $l\left(a^{\infty}, 1\right)$. In $X^{\prime}$, $\left(a^{n} c^{n} \odot x_{0}^{\prime}\right)$ also converges to $l\left(a^{\infty}, 1\right)$, but $\left(a^{n} b^{n^{2}} c^{n^{2}} \odot x_{0}\right)$ converges to $l\left(a^{\infty}, 1 / 2\right)$. With $\zeta=l\left(a^{\infty}, 1\right)$, define $\Lambda^{\prime}(\zeta)$ as before, but replacing $G * X$ with $G \odot X^{\prime}$. Then again we see that $\Lambda^{\prime}(\zeta)$ is an interval in $\partial X^{\prime}$.

In fact, it follows from Theorem 2 that whenever $\mathbb{F}_{2} \times \mathbb{Z}$ acts geometrically on two $\operatorname{CAT}(0)$ spaces $X$ and $X^{\prime}$ and $\zeta \in \partial X$, then $\Lambda(\zeta)$ is either a point or an interval. In the above examples, one moves between the endpoints of this interval by controlling the ratio of $b$ 's to $a$ 's in the sequence $\left(g_{n}\right)$. Our final example shows that moving between the endpoints of this interval need not be so simple in general.

Example 2.3 (Diamonds). Begin with the diamond in $\mathbb{E}^{2}$ with vertices $\{(1,0),(0,-1),(-1,0),(0,1)\}$ (that is, the convex hull of these four points). Let $\bar{Q}$ be the space formed by gluing opposite vertices: $(0,1)$ to $(0,-1)$ and $(1,0)$ to $(-1,0)$. Then $\pi_{1}(\bar{Q})=\mathbb{F}_{2}$. Its universal cover $Q$ consists of diamonds glued vertex to vertex with its fundamental group, $\mathbb{F}_{2}$, acting geometrically by deck transformations. The generators of $\mathbb{F}_{2}$ are represented by the 2 geodesic loops based at $(0,0)$ of length 2 which pass through the glued points.

We will use the symbol $\diamond$ for our action. Let $q_{0} \in Q$ be a preimage of the origin in $\bar{Q}$. In this space, $a$ and $b$ both translate $q_{0}$ a distance of 2 . But the geodesic $\left[q_{0}, a b \diamond q_{0}\right]$ is shorter than expected. More generally:

$$
d\left(q_{0},(a b)^{n} q_{0}\right)=(2 n-1) \sqrt{2}+2 \sim n 2 \sqrt{2}
$$

Let $G$ act on $X^{\prime \prime}=Q \times \mathbb{R}$ via the corresponding product action, and let $x_{0}^{\prime \prime}=\left(q_{0}, 0\right)$. In $G \cdot X$, the sequences $\left(a^{n} c^{n} \cdot x_{0}\right),\left(a^{n} b^{n^{2}} c^{n^{2}} \cdot x_{0}\right)$, and $\left(a^{n}(a b)^{n^{2}} c^{2 n^{2}} \cdot x_{0}\right)$ all converge to $l\left(a^{\infty}, 1\right)$. But in $X^{\prime \prime}$, the sequences $\left(a^{n} c^{n} \diamond x_{0}^{\prime \prime}\right)$ and $\left(a^{n} b^{n^{2}} c^{n^{2}} \diamond x_{0}^{\prime \prime}\right)$ converge to $l\left(a^{\infty}, 1 / 2\right)$ whereas the sequence $\left(a^{n}(a b)^{n^{2}} c^{2 n^{2}} \diamond x_{0}^{\prime \prime}\right)$ converges to $l\left(a^{\infty}, 1 / \sqrt{2}\right)$. Again $\Lambda\left(a^{\infty}\right) \subset \partial X^{\prime \prime}$ is an interval. But we do not move between the endpoints by controlling the ratio of $a$ 's to $b$ 's - we move by controlling the number of subwords of the form $a b$ which appear. In other words, it is by "shuffling" the $a$ 's and $b$ 's that we maximize slope. 


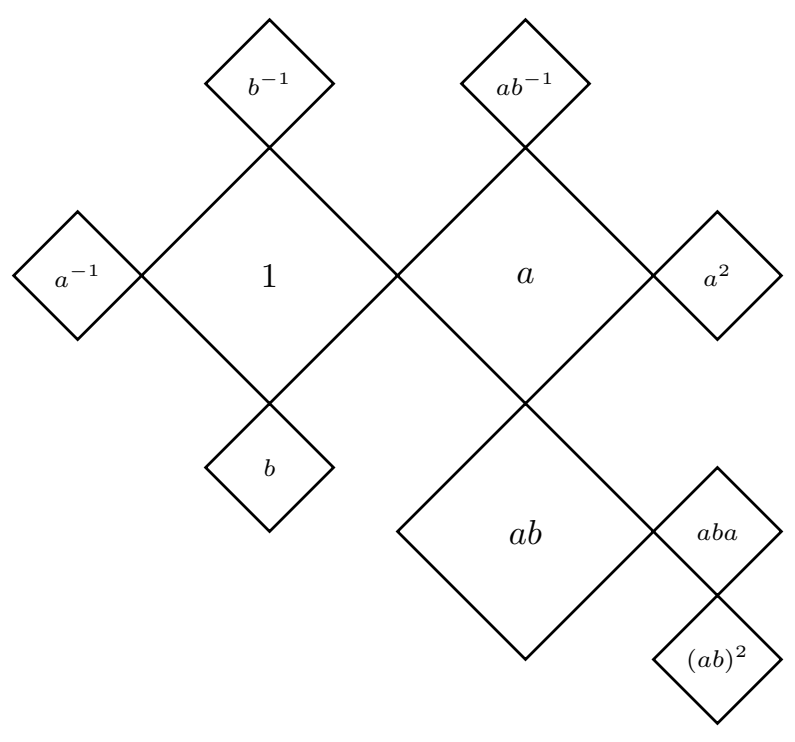

FiguRe $5 . Q$

2.2. Examples of Schmears. Assume $G$ acts geometrically on two CAT(0) spaces $X_{1}$ and $X_{2}$ with basepoints $x_{1} \in X_{1}$ and $x_{2} \in X_{2}$. Take $X=X_{1} \times X_{2}$ and $G^{\Delta}$ to be the "diagonal subgroup" described above. Given a group element $g \in G$, we denote the slope of the line segment $\left[x_{0}, g^{\Delta} x_{0}\right]$ in this splitting by $\operatorname{M}(g)$. Then $\partial X$ is the join of $\partial X_{1}$ with $\partial X_{2} . \Lambda=\operatorname{limset}\left(X, G^{\Delta}\right)$ lives in the interiors of the join arcs. The schmear maps $\Lambda \rightarrow \partial X_{i}$ come from collapsing the join arcs onto $\partial X_{i}$.

Interestingly, even though all boundaries of a negatively curved group $G$ are equivalent and the schmears of these boundaries are typically homeomorphic to $\partial G \times I$ for some interval $I$, these schmears need not be equivalent themselves. In fact there need not exist limset maps going in either direction, as exhibited here:

Example 2.4 (Two Schmears for Boundaries of $\mathbb{F}_{2}$ ). Using the notation given in Example2.2 (the stretching example), take $G=\mathbb{F}_{2}, X_{1}=\Gamma$, and $X_{2}=\Gamma^{\prime}$. Then the schmear of the pair $\partial X_{1}, \partial X_{2}$ is $\Lambda_{\odot}=\partial \mathbb{F}_{2} \times[1,2]$. For instance,

whereas

$$
\lim _{n \rightarrow \infty} \mathrm{M}_{\odot}\left(a^{n}\right)=1
$$

$$
\lim _{n \rightarrow \infty} \mathrm{M}_{\odot}\left(a^{n} b^{n^{2}}\right)=2,
$$

even though in $\mathbb{F}_{2} \cup \partial \mathbb{F}_{2}$, both sequences converge to $a^{\infty}$. The fact that $\mathbb{F}_{2}$ is strongly rigid means that the maps $\Lambda_{\odot} \rightarrow \partial X_{i}$ simply collapse the interval factors.

Now take $G=\mathbb{F}_{2}$ and $X_{1}=\Gamma$ but $X_{2}=Q$ from Example 2.3 (the diamonds example). Here the schmear is $\Lambda_{\diamond}=\partial \mathbb{F}_{2} \times[1 / \sqrt{2}, 1 / 2]$. It is homeomorphic to $\Lambda_{\odot}$, but not equivalent to it. In fact there are no limset maps going in either direction. For, in $G^{\Delta} \cup \Lambda_{\odot}$, the sequences $\left(a^{n}\right)^{\Delta}$ and $\left(a^{n} b^{n^{2}}\right)^{\Delta}$ converge to different points, whereas in $G^{\Delta} \cup \Lambda_{\diamond}$ they converge to the same point:

$$
\lim _{n \rightarrow \infty} \mathrm{M}_{\diamond}\left(a^{n}\right)=\lim _{n \rightarrow \infty} \mathrm{M}_{\diamond}\left(a^{n} b^{n^{2}}\right)=\frac{1}{2} .
$$

On the other hand, the pair of sequences $\left(a^{n+n^{2}} b^{n^{2}}\right)^{\Delta}$ and $\left(a^{n}(a b)^{n^{2}}\right)^{\Delta}$ converge to different points of $\Lambda_{\diamond}$; the first has slope $1 / 2$, the second $1 / \sqrt{2}$. But they converge to the same point of $\Lambda_{\odot}$, since they are written using the exact same letters and shuffling of letters does not change $M_{\odot}$.

There is a bound on the Lebesgue convering dimension of a schmear in terms of the covering dimension of a boundary. Extending work of Bestvina [1, Geoghegan and Ontaneda have proven that the dimension of a group boundary is one less than the cohomological dimension of the group [9. So if the cohomological dimension of a $\operatorname{CAT}(0)$ group $G$ is $d+1$ (so that all of its boundaries have dimension $d$ ), then whenever $\Lambda$ 
is a schmear for a pair of boundaries $\partial X, \partial Y$, it lives in the space $\partial X \times \partial Y \times(0, \infty)$, which has dimension bounded by $2 d+1$.

The schmear maps $\phi_{i}: \Lambda \rightarrow \partial X_{i}$ can be taken to be the projection maps to the respective coordinates. So point preimages live in subspaces of the form

$$
\left\{\zeta_{1}\right\} \times \partial X_{2} \times(0, \infty)
$$

which has dimension $d+1$. For $\mathbb{F}_{2} \times \mathbb{Z}$, the bound on the dimension of the schmear is 3 , and the preimage of every point has dimension no more than 2 . The schmears of the pairs of boundaries for $\mathbb{F}_{2} \times \mathbb{Z}$ described in the previous subsection all have dimension 2. The following schmear has full dimension.

Example 2.5 (A 3-Dimensional Schmear for Boundaries of $\mathbb{F}_{2} \times \mathbb{Z}$ ). Let $\cdot$ denote the untwisted product action of $\mathbb{F}_{2} \times \mathbb{Z}$ on $X_{1}=\Gamma \times \mathbb{R}$ first described in Example 2.1 with $x_{1} \in X_{1}$ the preferred basepoint. Let also $\diamond$ denote the action of $\mathbb{F}_{2}$ on $Q$ first described in Example 2.3. Take $X_{2}=Q \times \mathbb{R}, v=(0,0)$, and $x_{2}=(v, 0)$ but with the following action by $\mathbb{F}_{2} \times \mathbb{Z}$ obtained by adding a twist to the product action:

$$
\begin{aligned}
a \star(v, t) & =(a \diamond v, t), \\
b \star(v, t) & =(b \diamond v, t+1), \\
\text { and } c \star(v, t) & =(v, t+1) .
\end{aligned}
$$

Here are four sequences which converge to $a^{\infty} \in \partial X_{1}$ but which "fan out" in both the $\partial X_{2}$ direction and the $(0, \infty)$ direction of the ambient space containing the schmear.

\begin{tabular}{|l|l|l|}
\hline$\left(g_{n}\right)$ & $\lim _{n \rightarrow \infty}\left(g_{n} \star x_{2}\right)$ & $\lim _{n \rightarrow \infty} \mathrm{M}\left(g_{n}\right)$ \\
\hline \hline$x_{n}=a^{n} b^{-n^{2}}$ & $l\left(a^{\infty},-\frac{1}{2}\right)$ & $\sqrt{5}$ \\
\hline$y_{n}=a^{n} b^{n^{2}}$ & $l\left(a^{\infty}, \frac{1}{2}\right)$ & $\sqrt{5}$ \\
\hline$x_{n}^{\prime}=a^{n}\left(a b^{-1}\right)^{n^{2}}$ & $l\left(a^{\infty},-\frac{1}{\sqrt{2}}\right)$ & 3 \\
\hline$y_{n}^{\prime}=a^{n}(a b)^{n^{2}}$ & $l\left(a^{\infty}, \frac{1}{\sqrt{2}}\right)$ & 3 \\
\hline
\end{tabular}

It follows from Theorem 2 that the preimage of $a^{\infty}$ under the schmear map $\Lambda \rightarrow \partial X_{1}$ contains the convex hull of these four points and is a 2-disk $D^{2}$. The entire schmear is homeomorphic to the suspension of $C \times D^{2}$

\section{Preliminaries}

A compactification of a Hausdorff space $X$ is a topological embedding $X \hookrightarrow \bar{X}$ into a compact Hausdorff space whose image is dense. We will refer to $\bar{X}-X$ as a limset for $X$. Let $\Lambda, \Lambda^{\prime}$ be limsets of a noncompact space $X$. We write $\Lambda \geq \Lambda^{\prime}$ if the identity function $X \rightarrow X$ extends continuously to a map $X \cup \Lambda \rightarrow X \cup \Lambda^{\prime}$. The restriction of this map to $\Lambda \rightarrow \Lambda^{\prime}$ is called a limset map. As mentioned above, if this map is a homeomorphism, then $\Lambda$ and $\Lambda^{\prime}$ are considered equivalent. Let $X$ be a noncompact space and $\mathcal{L}(X)$ denote the collection of equivalence classes of limsets.

Remark 3.1. The reader should note that homeomorphic or even isometric limsets may represent different elements of $\mathcal{L}(X)$. When we refer to $\Lambda$ as an element of $\mathcal{L}(X)$, a particular compactification (informally, the way $\Lambda$ is glued to $X)$ is assumed.

The following statements are easily verified.

Lemma 3.2. Let $X$ be a noncompact Hausdorff space $X$. For $\Lambda, \Lambda^{\prime} \in \mathcal{L}(X), \Lambda \geq \Lambda^{\prime}$ iff the following rule holds: Given any sequence $\left(x_{n}\right) \subset X$ converging to a point of $\Lambda,\left(x_{n}\right)$ also converges to a point of $\Lambda^{\prime}$.

Lemma 3.3. Let $X$ be a noncompact Hausdorff space. Then

(1) Limset maps between elements of $\mathcal{L}(X)$ are unique and surjective (when they exist)

(2) $\geq$ is a partial ordering on $\mathcal{L}(X)$

(3) Every diagram of limset maps commutes 
Remark 3.4. If $X$ is a noncompact Hausdorff space, then $\mathcal{L}(X)$ has a unique maximum, namely $\beta X-X$ where $\beta X$ is the Stone-Čech compactification of $X$.

Lemma 3.5. Let $Y \subset X$ be a subspace, $\Lambda \geq \Lambda^{\prime}$ be limsets of $X$. Let $\Lambda(Y)=\bar{Y} \cap \Lambda$ and $\Lambda^{\prime}(Y)=\bar{Y} \cap \Lambda^{\prime}$. Then $\Lambda(Y) \geq \Lambda^{\prime}(Y)$ via the restriction of the limset map $\Lambda \rightarrow \Lambda^{\prime}$ to $\Lambda(Y)$.

3.1. Notes on CAT(0) Spaces and Groups. Let $(X, d)$ be a $\operatorname{CAT}(0)$ space. Given two geodesics $\alpha, \beta$ : $[0,1] \rightarrow X$ parameterized to have constant speed, the distance metric satisfies

$$
d(\alpha(t), \beta(t)) \leq t d(\alpha(1), \beta(1))+(1-t) d(\alpha(0), \beta(0)) .
$$

for all $t \in[0,1]$. This property is known as convexity of the metric.

For the most part we will consider the visual boundary as the set of all geodesic rays emanating from a common basepoint $x_{0} \in X$. With the cone topology, $\bar{X}=X \cup \partial X$ is a compactification of $X$. $\bar{X}$ can be identified with the space of geodesic segments and rays emanating from $x_{0}$ parameterized to have unit speed. Then the topology on $\bar{X}$ is the same as the compact-open topology on this function space.

Here is how we can tell if a sequence of points $\left(x_{n}\right)$ in $\bar{X}$ converges to a point $\zeta \in \partial X$. Let $\gamma_{n}$ and $\alpha$ be the geodesics based at $x_{0}$ determining $x_{n}$ and $\zeta$ respectively, and $\delta>0$ be arbitrary. Then $x_{n} \rightarrow \zeta$ iff for any $K \geq 0$, there is an $N \geq 0$ such that for all $n \geq N$, either $x_{n} \in \partial X$ or $d\left(x_{0}, x_{n}\right) \geq N$, and $d\left(\alpha(K), \gamma_{n}(K)\right) \leq \delta$. Convexity of the metric then guarantees that $\gamma_{n} \rightarrow \alpha$ uniformly on compact subsets of $\mathbb{R}$.

Let $G$ be a group acting on a proper CAT(0) space $X$ by isometries. The translation length of $g \in G$ is defined by $|g|=\inf _{x \in X} d(x, g x)$. If this value is realized then we call $g$ semi-simple. Proposition II.6.10 in [3] tells us that whenever a group acts geometrically on a proper CAT(0) space $X$, all of its elements are semi-simple. The set of all points on which this minimum is attained is called the minset of $g$ :

$$
\operatorname{Min}_{X}(g)=\operatorname{Min}(g)=\{x \in X|d(x, g x)=| g \mid\} .
$$

If $|g|>0$, then $g$ is called hyperbolic. Whenever $x$ is in the minset of a hyperbolic element $g$, then there is a geodesic line $A$ passing through $x$ which is $g$ invariant. This line is called an axis of $g$. For a subgroup $H \leq G$ of isometries of a CAT(0) space $X$ the minset of $H$ is defined as

$$
\operatorname{Min}_{X}(H)=\operatorname{Min}(H)=\bigcap_{g \in H} \operatorname{Min}(g) .
$$

By [3, Proposition II.6.2], minsets of isometries and minsets of groups of isometries are always closed and convex. If the ambient space is complete (as we will always assume), then minsets are themselves complete CAT(0) spaces.

A key ingredient in Theorem 2 is the Flat Torus Theorem [3, Theorem II.7.1]. Recall that an isometry $g$ of a product $X_{1} \times X_{2}$ is said to respect the product decomposition if $g$ can be written in coordinates as $g=\left(g_{1}, g_{2}\right)$ where $g_{1}$ and $g_{2}$ are isometries of $X_{1}$ and $X_{2}$.

Theorem (Flat Torus Theorem). Let $A=\mathbb{Z}^{d}$ act properly discontinuously by semi-simple isometries on a proper $C A T(0)$ space $X$. Then:

(1) $\operatorname{Min}(A)$ is nonempty and splits as a product $Y \times \mathbb{E}^{d}$.

(2) Every $c \in A$ leaves $\operatorname{Min}(A)$ invariant and respects the product decomposition; $c$ acts as the identity on $Y$ and as a translation on $\mathbb{E}^{d}$.

(3) The quotient of each flat $\{y\} \times \mathbb{E}^{d}$ by the action of $A$ is an $n$-torus.

(4) If an isometry of $X$ normalizes $A$, then it leaves $\operatorname{Min}(A)$ invariant and preserves the product decomposition.

Another fact about minsets which will come in handy is [3, Proposition II.6.9]: 
Proposition 3.6. Let $X_{1}$ and $X_{2}$ be proper $C A T(0)$ spaces and $g_{i}$ be isometries of $X_{i}$, and consider the isometry $g=\left(g_{1}, g_{2}\right)$ of $X=X_{1} \times X_{2}$. Then

$$
\operatorname{Min}_{X} g=\operatorname{Min}_{X_{1}}\left(g_{1}\right) \times \operatorname{Min}_{X_{2}}\left(g_{2}\right)
$$

In particular, $g$ is semi-simple iff both $g_{1}$ and $g_{2}$ are.

3.2. A Category of CAT(0) Limsets. If a group $G$ acts by isometries on a $\operatorname{CAT}(0)$ space $X$ in such a way that $G \rightarrow X$ is a QIE, we will say that $G$ acts pseudo-geometrically on $X$. The key way in which psuedogeometric actions arise in this paper is as follows: Whenever a group $G$ acts geometrically on a $\operatorname{CAT}(0)$ space $X$ and $H \leq G$ is a quasi-isometrically embedded subgroup, then the action of $H$ as a subgroup is pseudo-geometric.

We denote by $\mathcal{L}^{\mathrm{CAT}(0)}(G)$ the subcollection of $\mathcal{L}(G)$ consisting of limsets which come from pseudogeometric actions. Formally,

$$
\mathcal{L}^{\operatorname{CAT}(0)}(G)=\{\operatorname{limset}(X, G) \mid G \text { acts pseudo-geometrically on a } \operatorname{CAT}(0) \text { space } X\} / \sim
$$

where $\sim$ denotes the equivalence relation described above.

Remark 3.7. By Theorem 1 , $\mathcal{L}^{\mathrm{CAT}(0)}(G)$ is a directed poset which means that it has the structure of an inverse system.

Example 3.8 (Limset Maps for $\mathbb{Z}^{d}$ ). Whenever $\mathbb{Z}^{d}$ acts properly discontinuously by semi-simple isometries on a CAT(0) space $X$, the Flat Torus Theorem guarantees that it acts cocompactly on the convex hull of the orbit of a point. The point can be chosen so that this convex hull is $\mathbb{E}^{d}$, and $\operatorname{limset}\left(X, \mathbb{Z}^{d}\right)=\partial \mathbb{E}^{d}=S^{d-1}$. It follows that all limsets in $\mathcal{L}^{\mathrm{CAT}(0)}\left(\mathbb{Z}^{d}\right)$ which come from semi-simple isometries are equivalent.

This line of reasoning also gives

Corollary 3.9 (Corollary to the Flat Torus Theorem). Let $\mathbb{Z}^{d}$ act by semi-simple isometries on two CAT(0) spaces $X_{1}$ and $X_{2}$. Then there is a limset map $\operatorname{limset}\left(X_{1}, \mathbb{Z}^{d}\right) \rightarrow \operatorname{limset}\left(X_{2}, \mathbb{Z}^{d}\right)$ which is a homeomorphism.

Lemma 3.10. Let $\Lambda \geq \Lambda^{\prime} \in \mathcal{L}^{C A T(0)}(G)$ and $H \leq G$ be quasi-isometrically embedded subgroup. Denote by $\Lambda(H), \Lambda^{\prime}(H)$ the corresponding subsets which are the limsets of $H$. Then the limset map $\phi: \Lambda \rightarrow \Lambda^{\prime}$ restricts to a limset map $\phi_{H}: \Lambda(H) \rightarrow \Lambda^{\prime}(H)$.

In general, the preimage of $\Lambda^{\prime}(H)$ under the unrestricted map $\phi$ can be larger than $\Lambda(H)$.

Example 3.11. Consider Example 2.1. We have $\mathbb{F}_{2}$ acting on $\Gamma \times \mathbb{R}$ in two ways; let $X_{1}$ denote $\Gamma \times \mathbb{R}$ with the twisted action, $X_{2}=\Gamma$ with the standard action, and $H=\langle a\rangle$. There is a limset map $\phi: \operatorname{limset}\left(X_{1}, \mathbb{F}_{2}\right) \rightarrow$ $\operatorname{limset}\left(X_{2}, \mathbb{F}_{2}\right)$. But limset $\left(X_{1}, H\right)$ is just the two points $l\left(\left\{a^{ \pm \infty}\right\}, 0\right)$ whereas $\phi^{-1}\left(\operatorname{limset}\left(X_{2}, H\right)\right)$ consists of two intervals.

All CAT $(0)$ boundaries of $\mathbb{F}_{2}$ are equivalent in $\mathcal{L}^{\mathrm{CAT}(0)}\left(\mathbb{F}_{2}\right)$, since it is negatively curved. They are homeomorphic to the Cantor set $C$. But unless the actions chosen on the spaces $X_{1}$ and $X_{2}$ are very close, the schmears constructed will be homeomorphic to $C \times[0,1]$. But as mentioned in Example 2.4 these schmears need not themselves even be comparable in $\mathcal{L}^{\mathrm{CAT}(0)}\left(\mathbb{F}_{2}\right)$.

The following proposition tells us that all of the major results in this paper hold if they hold for a subgroup of finite index.

Proposition 3.12. Let $H$ be a finite index subgroup of a $C A T(0)$ group $G$. Assume $G$ acts pseudogeometrically on $C A T(0)$ spaces $X$ and $Y$ with limsets $\Lambda$ and $\Lambda^{\prime}$ respectively. Then $\Lambda=\operatorname{limset}(X, H)$, $\Lambda^{\prime}=\operatorname{limset}(Y, H)$, and $\Lambda \geq \Lambda^{\prime}$ in $\mathcal{L}^{C A T(0)}(G)$ iff $\Lambda \geq \Lambda^{\prime}$ in $\mathcal{L}^{C A T(0)}(H)$. If so, then the limset map $\Lambda \rightarrow \Lambda^{\prime}$ which comes from $G$ is the same as that which comes from $H$.

Proof. Choose basepoints $x \in X$ and $y \in Y$. Since $H$ has finite index in $G$, inclusion $H \rightarrow G$ is a quasiisometry, and hence $H \rightarrow X$ and $H \rightarrow Y$ are QIEs. Let $K \geq 0$ be a constant such that given $g \in G$, there is an $h \in H$ such that $d_{X}(g x, h x)$ and $d_{Y}(g y, h y)$ are both bounded by $K$. Then in particular, if $\left(g_{n}\right) \subset G$ converges to a point of $\partial X$, there is a sequence $\left(h_{n}\right) \subset H$ such that $d_{X}\left(g_{n} x, h_{n} x\right) \leq K$, so that $\left(h_{n} x\right)$ converges to the same point of $\partial X$. Since $d_{Y}\left(g_{n} y, h_{n} y\right) \leq K$ as well, we know that the sequence $\left(g_{n} y\right)$ converges iff $\left(h_{n} y\right)$ converges, and if they do, then they converge to the same point of $\partial Y$. 


\section{The Schmear}

We begin this section with a more formal treatment of slopes in products of CAT $(0)$ spaces. Let $X=$ $X_{1} \times X_{2}$ be a product of two proper CAT(0) spaces. A path is a map of an interval $I \subset \mathbb{R}$ into a space, where $I$ may either be a closed interval $[0, D]$ or a half-open interval $[0, \infty)$. Given a path $\alpha: I \rightarrow X$, we will write $\alpha=\left(\alpha_{1}, \alpha_{2}\right)$ to mean that for every $t$ in the domain of $\alpha, \alpha(t)=\left(\alpha_{1}(t), \alpha_{2}(t)\right)$. For us a path $\alpha$ will be called a geodesic if there is a number $\sigma \geq 0$ such that for every $s \neq t$ in the domain of $\alpha$, we have

$$
\frac{d(\alpha(s), \alpha(t))}{|s-t|}=\sigma .
$$

$\sigma$ is called the speed of $\alpha$. This may mean that $\alpha$ is a geodesic segment (a geodesic with domain of the form $[0, D]$ ), a geodesic ray (a geodesic with domain of the form $[0, \infty)$ ), or even perhaps a constant geodesic (a geodesic whose image is a point). If $\sigma=1$, we say that the geodesic has unit speed.

Let $\alpha=\left(\alpha_{1}, \alpha_{2}\right)$ be a nonconstant geodesic in $X$. We define the slope of $\alpha$ by

$$
\mathrm{M}(\alpha)=\frac{d_{2}\left(\alpha_{2}(s), \alpha_{2}(t)\right)}{d_{1}\left(\alpha_{1}(s), \alpha_{1}(t)\right)}
$$

for some $s \neq t$ in the domain of $\alpha$. By the following lemma $M(\alpha)$ is well-defined and independent of choice of parameterization. As before, we allow $\infty$ as a value for $\mathrm{M}$.

Lemma 4.1. Let $\alpha=\left(\alpha_{1}, \alpha_{2}\right)$ be a path in $X_{1} \times X_{2}$. Then $\alpha$ is a geodesic iff $\alpha_{1}$ and $\alpha_{2}$ are. If $\sigma_{1}$ and $\sigma_{2}$ are the speeds of $\alpha_{1}$ and $\alpha_{2}$, then $\mathrm{M}(\alpha)=\sigma_{2} / \sigma_{1}$ and the speed of $\alpha$ is $\sqrt{\sigma_{1}^{2}+\sigma_{2}^{2}}$.

Proof. The fact that $\alpha$ is a geodesic iff each $\alpha_{i}$ is [3, Proposition I.5.3(3)]. Once this is established, we know that every geodesic $\alpha$ lives in a subspace isometric to a flat quadrant or square, where the equations relating speeds and slope are easily verified.

In the current setup if $\alpha_{1}$ is constant (its image is just a point), then it is natural to say that $\mathrm{M}(\alpha)=\infty$. If we fix a basepoint $x_{0} \in X$ and identify points of $X \cup \partial X$ with the collection of unit speed geodesics emanating from $x_{0}$, then this gives a function

$$
\mathrm{M}:\left(X-\left\{x_{0}\right\}\right) \cup \partial X \rightarrow[0, \infty] .
$$

Proposition 4.2. $\mathrm{M}$ is continuous.

Proof. Fix $\epsilon>0$ and let $\pi_{\epsilon}$ denote the geodesic retraction based at $x_{0}$

$$
X \cup \partial X-B_{\epsilon}\left(x_{0}\right) \rightarrow S_{\epsilon}\left(x_{0}\right)
$$

where $S_{\epsilon}$ denotes the sphere of radius $\epsilon$; this is a continuous function by convexity of the metric. Let $\rho_{i}$ denote coordinate projection $X \rightarrow X_{i}$, which is also continuous. Finally, let $\delta_{i}(x)=d_{i}\left(\rho_{i}\left(x_{i}\right), \rho_{i}\left(x_{0}\right)\right)$. Then on $X \cup \partial X-B_{\epsilon}\left(x_{0}\right)$,

$$
\mathrm{M}=\frac{\delta_{2} \circ \pi_{\epsilon}}{\delta_{1} \circ \pi_{\epsilon}} .
$$

Therefore $\mathrm{M}$ is continuous on $X \cup \partial X-B_{\epsilon}\left(x_{0}\right)$ for every $\epsilon>0$.

If $G$ acts properly discontinuously on $X$, then for $g \in G$ not stabilizing $x_{0}$, we may define $\mathrm{M}(g)=$ $\mathrm{M}\left(\left[x_{0}, g x_{0}\right]\right)$. Since the stabilizer of $x_{0}$ is finite and we are interested in what happens at infinity, we will make the arbitrary definition $\mathrm{M}(g)=0$ when $g x_{0}=x_{0}$. M extends continuously to $\Lambda=\operatorname{limset}(X, G)$.

4.1. The Construction. This subsection completes the proof of Theorem 1 . Assume that $G$ acts pseudogeometrically on two CAT(0) spaces $X_{1}$ and $X_{2}$. Consider the inclusion $G \hookrightarrow G \times G$ as the diagonal subgroup $G^{\Delta}=\{(g, g) \mid g \in G\}$. We define

$$
\Lambda=\Lambda\left(G^{\Delta}, X_{1}, X_{2}\right)=\operatorname{limset}\left(X, G^{\Delta}\right) .
$$

Choose basepoints $x_{i} \in X_{i}$ and set $x=\left(x_{1}, x_{2}\right) \in X=X_{1} \times X_{2}$.

Lemma 4.3. The map $G^{\Delta} \rightarrow X$ is a QIE. If in addition the action of $G$ on each $X_{i}$ is semi-simple, then so is the action of $G^{\Delta}$ on $X$. 
Proof. Since $G \times G$ acts on $X$ geometricaly, we know that $G \times G \rightarrow X$ is a QIE by the Švarc-Milnor Lemma. To see that $G^{\Delta} \rightarrow X$ is a QIE it suffices to check that the isomorphic embedding $G \rightarrow G \times G$ which takes $G$ to $G^{\Delta}$ is a QIE. Let $l$ be a length metric on $G$ with respect to some finite generating set $\mathcal{S}$. Let $\mathcal{S}_{1}=\mathcal{S} \times\{1\}$ and $\mathcal{S}_{2}=\{1\} \times \mathcal{S}$ which generate $G \times\{1\}$ and $\{1\} \times G$ respectively. Then $\mathcal{S}_{1} \cup \mathcal{S}_{2}$ is a finite generating set for $G \times G$ inducing the length metric $l^{\prime}(g, h)=l(g)+l(h)$. In particular, $l^{\prime}(g, g)=2 l(g)$. To get the second statement, simply apply Proposition 3.6 .

Assume $G$ acts pseudo-geometrically on CAT(0) spaces $X_{1}$ and $X_{2}$ and consider the action of $G^{\Delta}$ on $X=X_{1} \times X_{2}$ with $\Lambda=\operatorname{limset}\left(X, G^{\Delta}\right)$.

Lemma 4.4. $\Lambda$ misses $\partial X_{1} \cup \partial X_{2}$.

Proof. We use the fact that the map $G x_{1} \rightarrow G x_{2}$ is a QIE to prove that $\mathrm{M}(\Lambda)$ is bounded away from 0 and $\infty$. Let $\lambda \geq 1$ and $\epsilon \geq 0$ be constants such that for $g \in G$,

$$
\frac{1}{\lambda} d_{1}\left(x_{1}, g x_{1}\right)-\epsilon \leq d_{2}\left(x_{2}, g x_{2}\right) \leq \lambda d_{1}\left(x_{1}, g x_{1}\right)+\epsilon .
$$

Then whenever we have a sequence $\left(g_{n}\right) \subset G$ such that $g_{n}^{\Delta} x \rightarrow \zeta \in \partial X$, it follows that $d_{1}\left(x_{1}, g_{n} x_{1}\right) \rightarrow \infty$. So

Letting $n \rightarrow \infty$, we get

$$
\frac{\frac{1}{\lambda} d_{1}\left(x_{1}, g_{n} x_{1}\right)-\epsilon}{d_{1}\left(x_{1}, g_{n} x_{1}\right)} \leq \mathrm{M}\left(g_{n}^{\Delta}\right) \leq \frac{\lambda d_{1}\left(x_{1}, g_{n} x_{1}\right)+\epsilon}{d_{1}\left(x_{1}, g_{n} x_{1}\right)} .
$$

$$
\frac{1}{\lambda} \leq \mathrm{M}(\zeta) \leq \lambda
$$

Consider the maps $e_{i}: \partial X-\left(\partial X_{1} \cup \partial X_{2}\right) \rightarrow \partial X_{i}$ which collapse the join arcs. Specifically, take a ray $\alpha=\left(\alpha_{1}, \alpha_{2}\right)$ in $X$ based at $x$. To say that $\alpha(\infty)$ lies interior to a join arc means that $\mathrm{M}(\alpha)$ is not 0 or $\infty$. Then $e_{i}(\alpha(\infty))=\alpha_{i}(\infty)$. This extends the projection map $X \rightarrow X_{i}$ to an (open) subset of $\partial X$. We take $\phi_{i}=\left.e_{i}\right|_{\Lambda}$. The following lemma completes the proof of Theorem 1

Lemma 4.5. $\phi_{1}$ and $\phi_{2}$ are limset maps.

Proof. Assume we have $\left(g_{n}\right) \subset G$ such that $g_{n}^{\Delta} x \rightarrow \zeta \in \partial X$. Let $\alpha=\left(\alpha_{1}, \alpha_{2}\right)$ be the unit speed geodesic ray with $\alpha(\infty)=\zeta$. Then $\phi_{i}(\zeta)=\alpha_{i}(\infty)$. Let $\gamma_{n}=\left(\gamma_{n}^{1}, \gamma_{n}^{2}\right)$ be a unit speed parameterization of $\left[x, g_{n}^{\Delta} x\right]$. Since projections do not increase distance, we have for all $t \geq 0$ and $n$ large enough so that $d\left(x, g_{n}^{\Delta} x\right) \geq t$, $d\left(\gamma_{n}^{i}(t), \alpha_{i}(t)\right) \leq d\left(\gamma_{n}(t), \alpha(t)\right)$. Therefore, $\gamma_{n}(t) \rightarrow \alpha(t)$ implies that $\gamma_{n}^{i}(t) \rightarrow \alpha_{i}(t)$.

Recent work by Link on lattices in certain $\mathrm{CAT}(0)$ groups allows us to understand schmears of negatively curved groups [13]. Although Link's work applies to a wider class of CAT(0) groups, the following argument requires strong rigidity, which is is only known for a handful of types of groups.

Corollary 4. Let $G$ be a negatively curved group acting gometrically on two CAT(0) spaces $X_{1}$ and $X_{2}$, and $\Lambda$ be the schmear of the (equivalent) pair $\partial X_{1}, \partial X_{2}$. Then $\Lambda$ is homeomorphic to $\partial G \times I$ where $I$ is a closed possibly degenerate interval. The limset maps $\Lambda \rightarrow \partial X_{1}$ simply collapse the I coordinate.

Proof. If $G$ is elementary, then $G^{\Delta}$ is an infinite virtually cyclic group acting by semi-simple isometries on the CAT(0) space $X=X_{1} \times X_{2}$. Its limit set $\Lambda$ is just two points, namely the endpoints of the axis of the finite index infinite cyclic subgroup. Assume $G$ is non-elementary. Then, in the language of [13, it contains two independent regular axial isometries. By Lemma 4.4 we have $\Lambda \subset \partial X_{1} \times \partial X_{2} \times(0, \infty)$ and the maps $\phi_{1}$ and $\phi_{2}$ collapse the third coordinate. If we reparameterize the third coordinate according to angles made with $X_{1}$ instead of slopes, then $\Lambda \subset \partial X_{1} \times \partial X_{2} \times(0, \pi / 2)$.

By [13, Theorem B], $\Lambda$ splits as a product $F_{G} \times P_{G}$ where $F_{G} \subset \partial X_{1} \times \partial X_{2}$ and $P_{G} \subset(0, \pi / 2)$. Since $G$ is strongly rigid, the two boundaries $\partial X_{1}$ and $\partial X_{2}$ are equivalent. By commutativity of the diagram

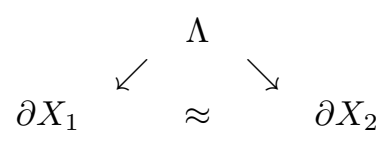

it follows that $F_{G}$ is the graph of the homeomorphism. By [13, Theorem $\left.\mathrm{C}\right], P_{G}$ is a closed interval. 
Recall that the Tits metric on the boundary of a CAT(0) space is the length metric associated to the Tits angle metric and generates a finer topology than the cone topology. In fact, there is a choice of $I \subset(0, \pi / 2)$ such that when $\partial G$ is given the Tits metric (giving it the topology of an uncountable discrete space) and $\Lambda$ is given the subspace metric corresponding to the Tits metric on $\partial\left(X_{1} \times X_{2}\right)$ (giving it the structure of a spherical join), then $I$ may be chosen so that the homeomorphism $\partial G \times I \rightarrow \Lambda$ is an isometry with respect to this metric.

Link's results apply in a much more general context then the negatively curved setting. For instance, the proof of this Corollary can be extended to groups $G$ acting geometrically on CAT(0) spaces with isolated flats. If they are not virtually abelian, then they have infinite Tits diameter by a theorem of Hruska and Kleiner in [11. Then we apply a theorem of Papasoglu and Swenson to find a pair of independent regular axial isometries [19].

\section{Geometric Actions of $G=H \times \mathbb{Z}^{d}$}

Let $H$ be a CAT(0) group and $G=H \times \mathbb{Z}^{d}$. We will write the coordinates of elements of $G$ in this direct product using the notation $\langle w, c\rangle$. Assume $G$ acts geometrically on a $\operatorname{CAT}(0)$ space $X$. Applying the Flat Torus Theorem, the minset $\operatorname{Min}\left(\mathbb{Z}^{d}\right)$ of $\mathbb{Z}^{d}$ is closed, convex, $G$-invariant, and splits as $Y \times E$ where $E$ is an isometric copy of $\mathbb{E}^{n}$. The fact that $\operatorname{Min}\left(\mathbb{Z}^{d}\right)$ is convex and $G$-invariant means that $\operatorname{limset}(X, G) \subset \partial \operatorname{Min}\left(\mathbb{Z}^{d}\right)$; for if we choose $x \in \operatorname{Min}\left(\mathbb{Z}^{d}\right)$, then $G x \subset \operatorname{Min}\left(\mathbb{Z}^{d}\right)$. We will assume that $X=Y \times E$. Furthermore, the action of $G$ preserves this splitting in the following sense: Every isometry $g \in G$ can be written coordinate-wise as $\left(g_{Y}, g_{E}\right)$ where $g_{Y}$ and $g_{E}$ are isometries of $Y$ and $E$. For clarity, the two notations are related by the rules $\langle w, c\rangle_{Y}=w_{Y}$ and $\langle w, c\rangle_{E}=w_{E} c_{E}$. The factor $\mathbb{Z}^{d}$ acts only in the $E$-coordinate; that is, for $c \in \mathbb{Z}^{d}$, $c_{Y}=\mathrm{id}_{Y}$. The action of $\mathbb{Z}^{d}$ on $E$ is geometric which means that $\partial E=\operatorname{limset}\left(X, \mathbb{Z}^{d}\right)$. In addition, the projected action of $H$ on $Y$ by the rule $h \mapsto h_{Y}$ is also geometric.

Choose a basepoint $y_{0} \in Y$ and let $x_{0}=\left(y_{0}, 0\right)$ be the specified basepoint in $X$ where 0 is the origin in $E$.

Lemma 5.1. The action of $H$ on $E$ is by translations.

Proof. Any isometry of $E$ which does not fix a point is a translation. Choose any $w \in H$. By hypothesis, $\mathbb{Z}^{d}$ centralizes $w_{E}$, and by (4) from the Flat Torus Theorem, Min $w_{E}$ is $\mathbb{Z}^{d}$-invariant. Since Min $w_{E}$ is convex, it follows that Min $w_{E}=E$. This means that if $w_{E}$ fixes a point, then $w_{E}=\mathrm{id}_{E}$.

5.1. A Slope Vector Function. For $g \in G$, we define the vertical translation of $g$ in $X$ to be the vector

$$
\overrightarrow{\mathrm{t}}(g)=g_{E} 0 \in E \text {. }
$$

We also define the horizontal displacement of $g$ by

$$
\mathrm{D}(g)=d_{Y}\left(y_{0}, g_{Y} y_{0}\right)
$$

Since $\mathbb{Z}^{d}$ acts only in the $E$-coordinate, $\mathrm{D}(\langle w, c\rangle)=\mathrm{D}(w)$. Let $\mathrm{M}$ denote the slope function based at $x_{0}$ with respect to the splitting $Y \times E$ and write $\bar{X}_{0}=\mathrm{M}^{-1}([0, \infty))$. This is just the complement of $E \cup \partial E$ in $X$. We define the slope vector map $\overrightarrow{\mathrm{m}}: \bar{X}_{0} \rightarrow E$ as follows. Identify $\bar{X}_{0}$ with the collection of nonvertical unit speed geodesics emanating from $x_{0}$. For such a geodesic $\gamma=(\alpha, \beta)$ in the domain, we define

$$
\overrightarrow{\mathrm{m}}(\gamma)=\frac{\beta(t)-\beta(s)}{d_{Y}(\alpha(t), \alpha(s))}
$$

where $s<t$ are in the domain of $\alpha$.

Lemma 5.2. $\overrightarrow{\mathrm{m}}$ is independent of which $s$ and $t$ are chosen and is continuous.

Proof. Let $\sigma_{\alpha}$ and $\sigma_{\beta}$ denote the speeds of $\alpha$ and $\beta$. Observe that

$$
\beta(t)-\beta(s)=(t-s) \overrightarrow{\mathrm{u}} \sigma_{\beta}
$$

where $\overrightarrow{\mathrm{u}} \in E$ is unit vector in the direction of $\beta$. Thus $\overrightarrow{\mathrm{m}}(\gamma)=\sigma_{\beta} \overrightarrow{\mathrm{u}} / \sigma_{\alpha}$, which is independent of $s$ and $t$. The proof that $\overrightarrow{\mathrm{m}}$ is continuous is the same as the proof of Proposition 4.2 but we replace $\delta_{2}$ with id $\mathrm{id}_{E}$.

Note that $\|\vec{m}\|$ is the same as the slope $M$ described earlier with respect to the current splitting where $\|\cdot\|$ denotes the standard Euclidean norm on $E$. 
Lemma 5.3. $\overrightarrow{\mathrm{m}}$ extends to a continuous map $\left(X-\left\{x_{0}\right\}\right) \cup \partial X \rightarrow E \cup \partial E$. Specifically, for $v \in E, \overrightarrow{\mathrm{m}}\left(y_{0}, v\right)$ is the geodesic ray emanating from 0 passing through $v$, and $\overrightarrow{\mathrm{m}}$ is the identity on $\partial E$.

Proof. For a point $x \in X \cup \partial X$ not in $E \cup \partial E$, define $\overrightarrow{\mathrm{u}}(x)=\overrightarrow{\mathrm{m}}(x) / \mathrm{M}(x)$. This is the unit vector in the direction of $\overrightarrow{\mathrm{m}}(x)$. If a sequence of points $\left(x_{n}\right) \subset \bar{X}_{0}$ converges to a point $z \in(E-0) \cup \partial E$, then eventually $\left(x_{n}\right)$ misses the subspace $Y \cup \partial Y$. Then $\overrightarrow{\mathrm{u}}\left(x_{n}\right) \rightarrow \overrightarrow{\mathrm{u}}(z)$ and $\mathrm{M}\left(x_{n}\right) \rightarrow \infty$. So $\overrightarrow{\mathrm{m}}\left(x_{n}\right)=\mathrm{M}\left(x_{n}\right) \overrightarrow{\mathrm{u}}\left(x_{n}\right)$ converges to the geodesic ray in the direction of $\overrightarrow{\mathrm{u}}(z)$.

As before, $\overrightarrow{\mathrm{m}}$ can be thought of as a continuous function of $G$ using the map $G \rightarrow G x_{0}$ provided we make the arbitrary definition $\overrightarrow{\mathrm{m}}(g)=0 \in E$ for $g$ stabilizing $x_{0}$. Note that for $g=\langle w, c\rangle \in G$ such that $\mathrm{D}(g)>0$, we have

$$
\overrightarrow{\mathrm{m}}(g)=\frac{\overrightarrow{\mathrm{t}}(g)}{\mathrm{D}(w)}=\frac{\overrightarrow{\mathrm{t}}(w)+\overrightarrow{\mathrm{t}}(c)}{\mathrm{D}(w)} .
$$

Ruane proves in 20] that whenever a $\operatorname{CAT}(0)$ group of the form $H \times \mathbb{Z}$ acts geometrically on a $\operatorname{CAT}(0)$ space, then the limset of $H$ is bounded away from the limset of $\mathbb{Z}$. Here is an analogous result in this setting.

Proposition 5.4. $\overrightarrow{\mathrm{m}}$ is bounded on $H$.

Proof. Choose any finite generating set for $H$ and let $l$ be the corresponding length metric. Let $\lambda \geq 1$ and $\epsilon \geq 0$ be such that for all $w \in H, \mathrm{D}(w) \geq l(w) / \lambda-\epsilon$. Let $M$ be the maximum of $\|\overrightarrow{\mathrm{t}}\|$ on a finite generating set for $H$. Then for $w \in H,\|\overrightarrow{\mathrm{t}}(w)\| \leq M l(w)$ and $\|\overrightarrow{\mathrm{m}}(w)\| \leq M \lambda+1$ when $l(w)$ is sufficiently large.

We close this section by recording for later use a technical lemma which describes sequences converging to points of $\partial E$.

Lemma 5.5. Let $\left(g_{n}\right) \subset G$ be a sequence such that $g_{n} x_{0}$ converges to a point of $\partial E$, say $g_{n}=\left\langle w_{n}, c_{n}\right\rangle$. Then

$$
\lim _{n \rightarrow \infty} \frac{d\left(x_{0},\left\langle 1, c_{n}\right\rangle x_{0}\right)}{d\left(x_{0},\left\langle w_{n}, 1\right\rangle x_{0}\right)}=\infty
$$

Proof. The triangle inequality gives

$$
\frac{\left\|\overrightarrow{\mathrm{t}}\left(c_{n}\right)\right\|}{\mathrm{D}\left(w_{n}\right)} \geq\left\|\overrightarrow{\mathrm{m}}\left(g_{n}\right)\right\|-\left\|\overrightarrow{\mathrm{m}}\left(w_{n}\right)\right\|
$$

The previous two results tell us that $\left\|\overrightarrow{\mathrm{m}}\left(g_{n}\right)\right\| \rightarrow \infty$ and that $\left\|\overrightarrow{\mathrm{m}}\left(w_{n}\right)\right\|$ is bounded. Therefore

$$
\frac{d\left(x_{0},\left\langle 1, c_{n}\right\rangle x_{0}\right)^{2}}{d\left(x_{0},\left\langle w_{n}, 1\right\rangle x_{0}\right)^{2}}=\frac{\left\|\overrightarrow{\mathrm{t}}\left(c_{n}\right)\right\|^{2}}{\mathrm{D}\left(w_{n}\right)^{2}} \cdot \frac{1}{\left\|\overrightarrow{\mathrm{m}}\left(w_{n}\right)\right\|^{2}+1} \rightarrow \infty .
$$

\section{Cell-Like Limset Maps}

Let $G=\mathbb{F}_{m} \times \mathbb{Z}^{d}$ where $\mathbb{F}_{m}$ is the free group on $m$ generators where $m \geq 2$. Assume $G$ acts geometrically on a proper CAT(0) space $X$. The standard generating set for $G$ is the union of the standard basis for $\mathbb{Z}^{d}$ along with $m$ generators for $\mathbb{F}_{m}$. The corresponding length metric on $G$ satisfies $l_{G}(\langle w, c\rangle)=l_{\mathbb{F}_{m}}(w)+l_{\mathbb{Z}^{d}}(c)$. Our proof of Theorem 2 here relies heavily on the fact that $\mathbb{F}_{m}$ is negatively curved.

6.1. Straight Elements. Denote $l=l_{\mathbb{F}_{m}}$. An element $w \in \mathbb{F}_{m}$ is called straight if it satisfies $l\left(w^{2}\right)=2 l(w)$. Let $\Gamma$ denote the Cayley graph of $\mathbb{F}_{m}$ with respect to the standard generating set, an infinite $2 m$-valent tree. Recall that a subspace $A$ of a metric space $B$ is called quasi-dense or $C$-dense if there is a $C \geq 0$ such that $B$ is contained in the $C$-neighborhood of $A$.

Lemma 6.1. The following are equivalent for $w \in \mathbb{F}_{m}$ :

(1) $w$ is straight.

(2) If $a_{1} \ldots a_{n}$ is the unique reduced spelling for $w$, then $a_{n} \neq a_{1}^{-1}$. 
(3) In the action of $\mathbb{F}_{m}$ on $\Gamma, w$ has an axis passing through the identity.

The set of straight elements is 1-dense in $\mathbb{F}_{m}$ and powers of straight elements are straight.

Proof. Choose $w \in \mathbb{F}_{m}$ and let $a_{1} \ldots a_{n}$ be the reduced spelling for $w$. Saying that $w$ is straight means that the word $a_{1} \ldots a_{n} a_{1} \ldots a_{n}$ has no reductions. Since $\mathbb{F}_{m}$ is free, this means that $a_{n} \neq a_{1}^{-1}$. Thus (1) and (2) are equivalent. To see that (1) and (3) are equivalent simply note that $\Gamma$ is $\mathrm{CAT}(0)$ and the identity is in the minset of $w$ iff $w$ is straight.

Now we show that the set of straight elements is 1-dense. Suppose $w$ is not straight. Let $w=a_{1} \ldots a_{n}$ be a spelling in terms of the generating set. Since we assumed $m>1$, there is a letter $\epsilon$ which is neither $a_{1}$ nor its inverse. Since $a_{1}=a_{n}^{-1}, w \epsilon$ is straight.

Elements $\langle w, c\rangle \in G$ will be called straight if $w$ is straight. The reason for considering straight elements is the following.

Lemma 6.2. There is a compact set $D \subset X$ such that every straight element of $G$ has an axis in $X$ which passes through $D$.

Recall that quasi-geodesics in negatively curved spaces behave well [3, Theorem III.H.1.7]:

Theorem (Stability of Quasi-Geodesics). For all $\delta>0, \lambda \geq 1, \epsilon \geq 0$ there exists a constant $R=R(\delta, \lambda, \epsilon)$ with the following property:

If $X$ is a $\delta$-hyperbolic space, $c$ is a $(\lambda, \epsilon)$-quasi-geodesic in $X$ and $[p, q]$ is a geodesic segment joining the endpoints of $c$, then the Hausdorff distance between $[p, q]$ and the image of $c$ is less than $R$.

Proof of Lemma 6.2. Let $f: \Gamma \rightarrow Y$ denote the QIE. By the Stability of Quasi-Geodesics Theorem, there is an $R \geq 0$ such that for every geodesic $\gamma$ in $\Gamma, f(\gamma)$ tracks within a Hausdorff distance of $R$ from the geodesic in $Y$ between its endpoints. In particular, if $\gamma$ begins at a vertex $w$ and ends at a vertex $w^{\prime}$, then $f(\gamma)$ is within a Hausdorff distance of $R$ from $\left[y_{0}, w y_{0}\right]$. Now if $w$ is straight, then for every $n$ the geodesic in $\Gamma$ from $w^{-n}$ to $w^{n}$ passes through the identity. It follows that the geodesic $\left[w^{-n} y_{0}, w^{n} y_{0}\right]$ passes within a distance of $R$ from $y_{0}$. Letting $n \rightarrow \infty$, we see that every axis for $w$ passes through $K=\overline{B_{R}\left(y_{0}\right)}$. By Proposition 3.6 . if $z \in K$ is in the minset of $w$, then $(z, 0)$ is in the minset of $\langle w, c\rangle$ for any $c \in \mathbb{Z}^{d}$. Therefore every straight element of $G$ has an axis passing through $K \times\{0\}$.

Recall that two sequences of real numbers $\left(x_{n}\right)$ and $\left(y_{n}\right)$ are called asymptotic if their ratio $x_{n} / y_{n}$ converges to 1 as $n \rightarrow \infty$ and is written $x_{n} \sim y_{n}$.

Lemma 6.3. Let $\left(g_{n}\right) \subset G$ be a sequence of straight elements such that $g_{n} x_{0} \rightarrow \zeta \in \partial X$ and $\left(k_{n}\right)$ be any sequence of positive integers. Then $g_{n}^{k_{n}} x_{0} \rightarrow \zeta$ as well, and $d\left(x_{0}, g_{n}^{k_{n}} x_{0}\right) \sim k_{n} d\left(x_{0}, g_{n} x_{0}\right)$.

Proof. Let $\alpha$ be the geodesic ray based at $x_{0}$ going out to $\zeta$ and $R>0$ be large enough so that every straight element of $G$ has an axis passing through $B_{R}\left(x_{0}\right)$. For each $n$, let $x_{n} \in B_{n}\left(x_{0}, R\right)$ lie in the axis of $g_{n}$. Then the geodesics $\left[x_{0}, g_{n}^{k_{n}} x_{0}\right]$ and $\left[x_{0}, g_{n} x_{0}\right]$ both stay inside the $R$-tubular neighborhood of the axis of $g_{n}$ passing through $x_{n}$. It follows that $g_{n} x_{0}$ lies inside in the $2 R$-neighborhood of $\left[x_{0}, g_{n}^{k_{n}} x_{0}\right]$. Thus if $\left[x_{0}, g_{n} x_{0}\right]$ stays inside the 1 -neighborhood of $\alpha$ up to time $T$, then $\left[x_{0}, g_{n}^{k_{n}} x_{0}\right]$ stays inside the $(2 R+1)$-neighborhood of $\alpha$ up to time $T$. It follows that $g_{n}^{k_{n}} \rightarrow \zeta$.

Now denote $a_{n}=d\left(x_{0}, g_{n} x_{0}\right), a_{n}^{\prime}=d\left(x_{0}, g_{n}^{k_{n}} x_{0}\right), b_{n}=d\left(x_{n}, g_{n} x_{n}\right)$, and $b_{n}^{\prime}=d\left(x_{n}, g_{n}^{k_{n}} x_{n}\right)$. Then $b_{n} \leq a_{n} \leq b_{n}+2 R, b_{n}^{\prime} \leq a_{n}^{\prime} \leq b_{n}^{\prime}+2 R, b_{n}^{\prime}=k_{n} b_{n}$, and hence

$$
\frac{a_{n}^{\prime}}{a_{n}} \sim \frac{b_{n}^{\prime}}{b_{n}}=k_{n}
$$

Therefore $a_{n}^{\prime} \sim k_{n} a_{n}$ as desired.

6.2. Averaging Sequences. Let $G=\mathbb{F}_{m} \times \mathbb{Z}^{d}$ act geometrically on CAT(0) spaces $X_{1}$ and $X_{2}$. If $m=1$, then $G$ is free abelian and Theorem 2 follows from Corollary 3.9. So we will assume $m>1$. As before we may assume each space splits as $X_{i}=Y_{i} \times E_{i}$ where $E_{i}$ is an isometric copy of $\mathbb{E}^{d}$. Let $\overrightarrow{\mathrm{t}}_{i}$ and $\overrightarrow{\mathrm{m}}_{i}$ denote the vertical translation and slope vector functions for each $X_{i}$. We will denote horizontal displacement in $Y_{i}$ using $\mathrm{D}_{i}$. Let $y_{i}$ be chosen basepoints in $Y_{i}, x_{i}=\left(y_{i}, 0\right)$, and $x_{0}=\left(x_{1}, x_{2}\right)$. Given $g \in G$, we will let $\mathrm{M}(g)$ denote the slope of the line segment $\left[x_{0}, g^{\Delta} x_{0}\right]$ in terms of the splitting $X=X_{1} \times X_{2}$. 
A fourth piece of information we will need regards slopes in the product $Y_{1} \times Y_{2}$. Given $g \in G$ we consider the geodesic $\left[x_{0}, g^{\Delta} x_{0}\right]$ as living in $X=Y_{1} \times E_{1} \times Y_{2} \times E_{2}$ and take its coordinate projection $\gamma$ to $Y_{1} \times Y_{2}$. If $\gamma$ is nonconstant, it has a well defined slope which we denote by $\mathrm{N}(g)$. In other words,

$$
\mathrm{N}(g)=\frac{\mathrm{D}_{2}(g)}{\mathrm{D}_{1}(g)} .
$$

This makes sense as long as $g$ does not act only in the $E_{1} \times E_{2}$ coordinates. Since $\mathbb{F}_{m}$ is torsion free, this is the same as saying that when $g=\langle w, c\rangle$, then $w \neq 1$. Then $\mathbf{N}(g)=\mathrm{N}(w)$ which, by Lemma 4.4, is bounded away from 0 and $\infty$. $N$ extends continuously to $\partial X-\partial\left(E_{1} \times E_{2}\right)$.

Denote $\Lambda=\operatorname{limset}\left(X, G^{\Delta}\right)$ and $\Sigma=\operatorname{limset}\left(X,\left(\mathbb{Z}^{d}\right)^{\Delta}\right)$ and let $\phi_{i}: \Lambda \rightarrow \operatorname{limset}\left(X_{i}, G\right)$ denote the limset maps constructed in Section 4. The following lemma is needed in light of Example 3.11 .

Lemma 6.4. For each $i, \phi_{i}^{-1}\left(\partial E_{i}\right)=\Sigma$ and $\left.\phi_{i}\right|_{\partial E_{i}}$ is a homeomorphism.

Proof. By Lemma 3.10, it suffices to prove that $\phi_{i}^{-1}\left(\partial E_{i}\right) \subset \Sigma$ for each $i$. We will prove this for $i=1$. Choose $\zeta_{1} \in \partial E_{1}$, and $\zeta \in \phi_{1}^{-1}\left(\zeta_{1}\right)$. This means that there is a sequence $\left(g_{n}\right) \subset G$ such that $g_{n}^{\Delta} x_{0} \rightarrow \zeta$ and $g_{n} x_{1} \rightarrow \zeta_{1}$. Write $g_{n}=\left\langle w_{n}, c_{n}\right\rangle$. Let $\lambda \geq 1$ and $\epsilon \geq 0$ be such that the map $G x_{1} \rightarrow G x_{0}$ is a $(\lambda, \epsilon)$-QIE. Then

$$
\frac{d\left(x_{0},\left\langle 1, c_{n}\right\rangle^{\Delta} x_{0}\right)}{d\left(x_{0},\left\langle w_{n}, 1\right\rangle^{\Delta} x_{0}\right)} \geq \frac{\frac{1}{\lambda} d_{1}\left(x_{1},\left\langle 1, c_{n}\right\rangle x_{1}\right)-\epsilon}{\lambda d_{1}\left(x_{1},\left\langle w_{n}, 1\right\rangle x_{1}\right)+\epsilon} \rightarrow \infty
$$

by Lemma 5.5. Apply convexity of the metric to the geodesics $\left[x_{0},\left\langle 1, c_{n}\right\rangle^{\Delta} x_{0}\right]$ and $\left[x_{0},\left\langle w_{n}, c_{n}\right\rangle^{\Delta} x_{0}\right]$ to see that $\left\langle 1, c_{n}\right\rangle^{\Delta} x_{0}$ also converges to $\zeta$. So $\zeta \in \Sigma$, as desired. The second fact is Corollary 3.9

This lemma tells us that preimages of points in $\partial E_{i}$ are singletons, and therefore cell-like. Next we turn our attention to preimages of points not in $\partial E_{i}$. Now,

$$
\partial X-\partial X_{1} \approx\left(C^{o} \partial X_{1}\right) \times \partial X_{2}
$$

where $C^{o} \partial X_{1}$ denotes the open cone on $\partial X_{1}: C^{o} \partial X_{1}=\partial X_{1} *\left\{x_{0}\right\}-\partial X_{1}$. So for each $i$, we have

$$
\begin{aligned}
& \partial X_{i}-\partial E_{i} \approx \partial Y_{i} \times C^{o} \partial E_{i} \\
& \approx \partial Y_{i} \times E_{i} .
\end{aligned}
$$

The first coordinate of this homeomorphism is the extension of the projection map and the second coordinate is $\overrightarrow{\mathrm{m}}_{i} \circ \phi_{i}$. Define $\Lambda^{0}=\Lambda-\Sigma$, which, by the above reasoning, can be thought of as living in $\partial Y_{1} \times E_{1} \times \partial Y_{2} \times E_{2}$.

Proposition 6.5. The following diagram commutes:

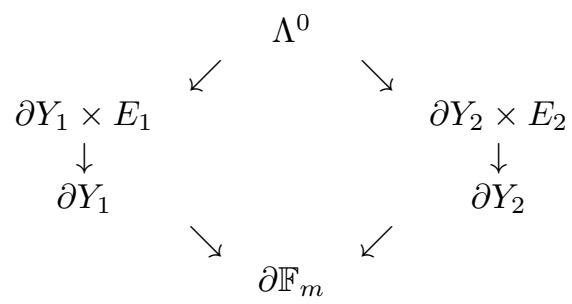

Proof. Suppose we have a sequence $\left(\left\langle w_{n}, c_{n}\right\rangle\right) \subset G$ such that $\left\langle w_{n}, c_{n}\right\rangle^{\Delta} x_{0} \rightarrow \zeta_{0} \in \Lambda^{0}$. Following the maps, we see that for both $i$, the sequences $\left(w_{n} y_{i}\right)$ converge to points $\zeta_{i} \in \partial Y_{i}$. By strong rigidity, the sequence $\left(w_{n}\right)$ converges to a point $\zeta_{0} \in \partial \mathbb{F}_{m}$. So for either $i, \zeta_{0} \in \Lambda^{0}$ gets mapped to $\zeta_{i} \in \partial Y_{i}$ which in turn gets mapped to $\zeta_{0} \in \partial \mathbb{F}_{m}$.

We now abuse notation by considering $\overrightarrow{\mathrm{m}}_{i}$ also as functions of $\Lambda^{0}$; that is, we write $\overrightarrow{\mathrm{m}}_{i}=\overrightarrow{\mathrm{m}}_{i} \phi_{i}$. Given $\eta \in \partial \mathbb{F}_{m}$, let $\Lambda_{\eta}$ denote its preimage in $\Lambda^{0}$ according to the diagram in the previous proposition.

Proposition 6.6. The map $\Lambda_{\eta} \rightarrow E_{1} \times E_{2} \times(0, \infty)$ given by $\left(\overrightarrow{\mathrm{m}}_{1}, \overrightarrow{\mathrm{m}}_{2}, 1 / \mathrm{N}\right)$ is an embedding and for each $i$, we have a commuting diagram

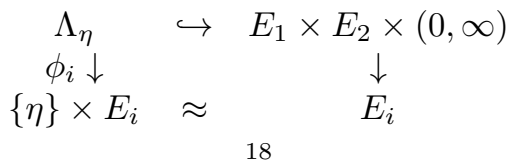


where the map down the right hand side is coordinate projection.

Proof. Since $\Lambda$ misses $\partial X_{1} \cup \partial X_{2}$, it lives in $\partial X_{1} \times \partial X_{2} \times(0, \infty)$. The last coordinate here is parameterized by $M$. Since the $\phi_{i}$ map $\Sigma$ homeomorphically onto $\partial E_{i}, \phi_{i}\left(\Lambda^{0}\right)$ lives in $\partial \mathbb{F}_{m} \times E_{i}$ where $E_{i}$ is parameterized by $\overrightarrow{\mathrm{m}}_{i}$. This means that $\Lambda^{0}$ lives in

$$
\partial \mathbb{F}_{m} \times E_{1} \times \partial \mathbb{F}_{m} \times E_{2} \times(0, \infty)
$$

Now by the previous proposition, the projection to each $\partial \mathbb{F}_{m}$ is just $\{\eta\}$. This means that the map

$$
\Lambda_{\eta} \rightarrow E_{1} \times E_{2} \times(0, \infty)
$$

given by $\left(\vec{m}_{1}, \vec{m}_{2}, M\right)$ is an embedding.

To change the third coordinate map to $1 / \mathrm{N}$, we just need to know that $\mathrm{M}$ can be written as a continuous function of $\overrightarrow{\mathrm{m}}_{1}, \overrightarrow{\mathrm{m}}_{2}$, and $\mathrm{N}$. Assume $\left(g_{n}\right) \subset G$ is a sequence such that $g_{n}^{\Delta} x_{0} \rightarrow \zeta \in \Lambda^{0}$. Then $\mathrm{D}_{i}\left(g_{n}\right)>0$ for large $n$ and we can write

$$
\mathrm{M}^{2}\left(g_{n}\right)=\frac{d_{2}\left(x_{2}, g_{n} x_{2}\right)^{2}}{d_{1}\left(x_{1}, g_{n} x_{1}\right)^{2}}=\mathrm{N}\left(g_{n}\right)^{2} \frac{\left\|\overrightarrow{\mathrm{m}}_{2}\left(g_{n}\right)\right\|^{2}+1}{\left\|\overrightarrow{\mathrm{m}}_{1}\left(g_{n}\right)\right\|^{2}+1}
$$

Letting $n \rightarrow \infty$, we get that

$$
M(\zeta)=N(\zeta) \sqrt{\frac{\left\|\vec{m}_{2}(\zeta)\right\|^{2}+1}{\left\|\vec{m}_{1}(\zeta)\right\|^{2}+1}}
$$

Lemma 6.7 (Sequence Averaging). Choose any $\zeta, \zeta^{\prime} \in \Lambda_{\eta}$ with the property that $\overrightarrow{\mathrm{m}}_{1}(\zeta)=\overrightarrow{\mathrm{m}}_{1}\left(\zeta^{\prime}\right)$. Then there are sequences $\left(a_{n}\right),\left(b_{n}\right),\left(c_{n}\right) \subset G$ such that $a_{n}^{\Delta} x_{0} \rightarrow \zeta, b_{n}^{\Delta} x_{0} \rightarrow \zeta^{\prime}$ and all of the following hold:

$$
\begin{aligned}
& \operatorname{limset}\left(X,\left\{c_{n}\right\}\right) \subset \Lambda_{\eta} \\
& \mathrm{D}_{1}\left(a_{n}\right)+\mathrm{D}_{1}\left(b_{n}\right) \sim \mathrm{D}_{1}\left(c_{n}\right) \\
& \mathrm{D}_{2}\left(a_{n}\right)+\mathrm{D}_{2}\left(b_{n}\right) \sim \mathrm{D}_{2}\left(c_{n}\right) \\
& \mathrm{D}_{2}\left(a_{n}\right) \sim \mathrm{D}_{2}\left(b_{n}\right)
\end{aligned}
$$

$$
\begin{aligned}
\overrightarrow{\mathrm{m}}_{1}\left(c_{n}\right) & \rightarrow \overrightarrow{\mathrm{m}}_{1}(\zeta) \\
\overrightarrow{\mathrm{m}}_{2}\left(c_{n}\right) & \rightarrow \frac{\overrightarrow{\mathrm{m}}_{2}(\zeta)+\overrightarrow{\mathrm{m}}_{2}\left(\zeta^{\prime}\right)}{2} \\
\mathrm{~N}\left(c_{n}\right)^{-1} & \rightarrow \frac{\mathrm{N}(\zeta)^{-1}+\mathrm{N}\left(\zeta^{\prime}\right)^{-1}}{2}
\end{aligned}
$$

Proof. Since the collection of straight elements is quasi-dense in $G$ and $G^{\Delta} \rightarrow X$ is a QIE, we can get sequences of straight elements $g_{n}=\left\langle v_{n}, \rho_{n}\right\rangle$ and $h_{n}=\left\langle w_{n}, \sigma_{n}\right\rangle$ such that $g_{n}^{\Delta} x_{0} \rightarrow \zeta$ and $h_{n}^{\Delta} x_{0} \rightarrow \zeta^{\prime}$. Since $\zeta, \zeta^{\prime} \notin \Sigma, \mathrm{D}_{2}\left(w_{n}\right)$ and $\mathrm{D}_{2}\left(v_{n}\right)$ both go to infinity. Define $s_{n}$ and $t_{n}$ to be the floors of $\mathrm{D}_{2}\left(w_{n}\right)$ and $\mathrm{D}_{2}\left(v_{n}\right)$.

We will take $a_{n}=g_{n}^{s_{n}}, b_{n}=h_{n}^{t_{n}}$, and $c_{n}=a_{n} b_{n}=\left\langle v_{n}^{s_{n}} w_{n}^{t_{n}}, \rho_{n}^{s_{n}} \sigma_{n}^{t_{n}}\right\rangle$. The first two converge to $\zeta$ and $\zeta^{\prime}$ by Lemma 6.3. Now, since $v_{n}$ and $w_{n}$ both converge to $\eta \in \partial \mathbb{F}_{m}$, reduced spellings for $v_{n}$ and $w_{n}$ share the same first letter $\epsilon$ when $n$ is large. Since they are straight, they do not end in $\epsilon^{-1}$. Using the fact that $\mathbb{F}_{m}$ is free, a geodesic edge path $\gamma_{n}$ from 1 to $v_{n}^{s_{n}} w_{n}^{t_{n}}$ in $\Gamma$ passes through $v_{n}$. Since $v_{n} \rightarrow \eta$, so does $v_{n}^{s_{n}} w_{n}^{t_{n}}$. This gives (0).

By the same reasoning, $\gamma_{n}$ also passes through the vertex $v_{n}^{s_{n}}$. Since the $Y_{i}$ are $\delta$-hyperbolic, it follows from the Stability of Quasi-Geodesics Theorem that there is an $R$ such that for both $i, v_{n}^{s_{n}} y_{i}$ is inside the $R$-neighborhood of $\left[y_{i}, v_{n}^{s_{n}} w_{n}^{t_{n}} y_{i}\right]$. By convexity of the CAT(0) metric, we get (11) and (2).

For (3), we apply Lemma 6.3

$$
\frac{\mathrm{D}_{2}\left(v_{n}^{s_{n}}\right)}{\mathrm{D}_{2}\left(w_{n}^{t_{n}}\right)} \sim \frac{s_{n} \mathrm{D}_{2}\left(v_{n}\right)}{t_{n} \mathrm{D}_{2}\left(w_{n}\right)} \rightarrow 1
$$

For (4) and (5) we will use the following equation which is easy to check:

$$
\overrightarrow{\mathrm{m}}_{i}\left(c_{n}\right)=\overrightarrow{\mathrm{m}}_{i}\left(a_{n}\right) \frac{\mathrm{D}_{i}\left(a_{n}\right)}{\mathrm{D}_{i}\left(c_{n}\right)}+\overrightarrow{\mathrm{m}}_{i}\left(b_{n}\right) \frac{\mathrm{D}_{i}\left(b_{n}\right)}{\mathrm{D}_{i}\left(c_{n}\right)} .
$$


If $i=1$, we use $(\dagger)$ to compute

$$
\begin{aligned}
\left|\overrightarrow{\mathrm{m}}_{1}\left(c_{n}\right)-\overrightarrow{\mathrm{m}}_{1}\left(b_{n}\right)\right| & =\left|\overrightarrow{\mathrm{m}}_{1}\left(a_{n}\right) \frac{\mathrm{D}_{1}\left(a_{n}\right)}{\mathrm{D}_{1}\left(c_{n}\right)}+\overrightarrow{\mathrm{m}}_{1}\left(b_{n}\right)\left(\frac{\mathrm{D}_{1}\left(b_{n}\right)}{\mathrm{D}_{1}\left(c_{n}\right)}-1\right)\right| \\
& \leq\left|\overrightarrow{\mathrm{m}}_{1}\left(a_{n}\right) \frac{\mathrm{D}_{1}\left(a_{n}\right)}{\mathrm{D}_{1}\left(c_{n}\right)}-\overrightarrow{\mathrm{m}}_{1}\left(b_{n}\right) \frac{\mathrm{D}_{1}\left(a_{n}\right)}{\mathrm{D}_{1}\left(c_{n}\right)}\right|+\left|\overrightarrow{\mathrm{m}}_{1}\left(b_{n}\right) \frac{\mathrm{D}_{1}\left(a_{n}\right)}{\mathrm{D}_{1}\left(c_{n}\right)}+\overrightarrow{\mathrm{m}}_{1}\left(b_{n}\right)\left(\frac{\mathrm{D}_{1}\left(b_{n}\right)}{\mathrm{D}_{1}\left(c_{n}\right)}-1\right)\right| \\
& =\frac{\mathrm{D}_{1}\left(a_{n}\right)}{\mathrm{D}_{1}\left(c_{n}\right)}\left|\overrightarrow{\mathrm{m}}_{1}\left(a_{n}\right)-\overrightarrow{\mathrm{m}}_{1}\left(b_{n}\right)\right|+\overrightarrow{\mathrm{m}}_{1}\left(b_{n}\right)\left|\frac{\mathrm{D}_{1}\left(a_{n}\right)+\mathrm{D}_{1}\left(b_{n}\right)}{\mathrm{D}_{1}\left(c_{n}\right)}-1\right| .
\end{aligned}
$$

Using (1) and the facts that $0 \leq \mathrm{D}_{1}\left(a_{n}\right) \leq \mathrm{D}_{1}\left(c_{n}\right)$ and $\overrightarrow{\mathrm{m}}_{1}\left(a_{n}\right)$ and $\overrightarrow{\mathrm{m}}_{1}\left(b_{n}\right)$ both converge to $\overrightarrow{\mathrm{m}}_{1}(\zeta)<\infty$, this all goes to zero as $n \rightarrow \infty$ giving (4).

Using (2) and (3) we get

$$
\frac{\mathrm{D}_{2}\left(a_{n}\right)}{\mathrm{D}_{2}\left(c_{n}\right)} \sim \frac{\mathrm{D}_{2}\left(b_{n}\right)}{\mathrm{D}_{2}\left(c_{n}\right)} \rightarrow \frac{1}{2}
$$

Apply ( $\dagger$ ) and let $n \rightarrow \infty$ to get (5).

Finally, we use (1), (2), and (3) to compute (6):

$$
\begin{aligned}
\mathrm{N}\left(c_{n}\right)^{-1} & \sim \frac{\frac{\mathrm{D}_{2}\left(a_{n}\right)}{\mathrm{D}_{2}\left(b_{n}\right)} \mathrm{N}\left(a_{n}\right)^{-1}+\mathrm{N}\left(b_{n}\right)^{-1}}{\frac{\mathrm{D}_{2}\left(a_{n}\right)}{\mathrm{D}_{2}\left(b_{n}\right)}+1} \\
& \rightarrow \frac{\mathrm{N}(\zeta)^{-1}+\mathrm{N}\left(\zeta^{\prime}\right)^{-1}}{2} .
\end{aligned}
$$

Proof of Theorem 2. By Lemma 6.4 we know that preimages of points of $\partial E_{1}$ are just points. It remains to consider preimages of points of $\partial X_{1}-\partial E_{1}$. Choose $\eta \in \partial \mathbb{F}_{m}$. Proposition 6.6 provided an embedding $\Lambda_{\eta} \hookrightarrow E_{1} \times E_{2} \times(0, \infty) \subset \mathbb{E}^{2 d+1}$ by the map $\left(\overrightarrow{\mathrm{m}}_{1}, \overrightarrow{\mathrm{m}}_{2}, \mathrm{~N}^{-1}\right)$. Choose any $\zeta_{1} \in \phi_{1}\left(\Lambda_{\eta}\right)$ and $\zeta, \zeta^{\prime} \in \phi_{1}^{-1}\left(\zeta_{1}\right)$. This means that $\overrightarrow{\mathrm{m}}_{1}(\zeta)=\overrightarrow{\mathrm{m}}_{1}\left(\zeta^{\prime}\right)=\overrightarrow{\mathrm{m}}_{1}\left(\zeta_{1}\right)$.

Get sequences $\left(a_{n}\right),\left(b_{n}\right),\left(c_{n}\right) \subset G$ such that $a_{n}^{\Delta} x_{0} \rightarrow \zeta$ and $b_{n}^{\Delta} x_{0} \rightarrow \zeta^{\prime}$, as prescribed by the previous lemma. From (10), (40), (5), and (6) it follows that $\left(c_{n}\right)$ converges to a point $\zeta^{\prime \prime} \in \Lambda_{\eta}$. (44) tells us that $\zeta^{\prime \prime} \in \phi_{1}^{-1}\left(\zeta_{1}\right)$ and (5) and (6) tell us that $\zeta^{\prime \prime}$ is actually the midpoint of the line segment $\left[\zeta, \zeta^{\prime}\right]$. By applying this averaging process repeatedly, we see that a dense subset of $\left[\zeta, \zeta^{\prime}\right]$ is contained in $\phi_{1}^{-1}\left(\zeta_{1}\right)$. But of course $\phi_{1}^{-1}\left(\zeta_{1}\right)$ is closed, which means that $\left[\zeta, \zeta^{\prime}\right] \subset \phi_{1}^{-1}\left(\zeta_{1}\right)$. This proves that $\phi_{1}^{-1}\left(\zeta_{1}\right)$ is a convex subspace of $\mathbb{E}^{2 d+1}$. Convex subspaces of euclidean spaces are disks. The proof for $\phi_{2}$ is the same.

Proof of Theorem 3. We need to prove that the only limset maps between boundaries of $\mathbb{F}_{2} \times \mathbb{Z}$ are homeomorphisms. Assume $G=\mathbb{F}_{2} \times \mathbb{Z}$ acts geometrically on two CAT(0) spaces $X_{1}$ and $X_{2}$ with specified basepoints $x_{1}$ and $x_{2}$ and that we have a limset map $\rho: \partial X_{1} \rightarrow \partial X_{2}$ which is not a homeomorphism. If $\rho$ were injective, then by Lemma 3.2, $\rho$ would be a homeomorphism, giving us a contradiction.

So $\rho$ must not be injective. It follows from Lemma 6.4 that $\rho$ takes the poles of $\partial X_{1}$ to the poles of $\partial X_{2}$, and from Proposition 6.5 that $\rho$ takes longitudes to longitudes. So there are distinct $\zeta_{1}, \zeta_{1}^{\prime} \in \partial X_{1}$ lying in the same longitude such that $\rho\left(\zeta_{1}\right)=\rho\left(\zeta_{1}^{\prime}\right)$; call this point $\zeta_{2}$. Let $c$ be the generator of $\mathbb{Z}$ and assume our coordinate system has been taken so that $\overrightarrow{\mathrm{t}}_{1}(c)$ and $\overrightarrow{\mathrm{t}}_{2}(c)$ are both positive. Choose sequences $\left(g_{n}\right),\left(g_{n}^{\prime}\right) \subset G$ such that $\left(g_{n} x_{1}\right)$ and $\left(g_{n}^{\prime} x_{1}\right)$ converge to $\zeta_{1}$ and $\zeta_{1}^{\prime}$, say $g_{n}=\left\langle w_{n}, c^{k_{n}}\right\rangle$ and $g_{n}^{\prime}=\left\langle w_{n}^{\prime}, c^{k_{n}^{\prime}}\right\rangle$.

Now the slopes and vertical displacements here are 1-dimensional vectors and, on these sequences, realvalued. To emphasize this, we will drop the vector notation and call them simply $\mathrm{m}_{1}, \mathrm{~m}_{2}, \mathrm{t}_{1}$, and $\mathrm{t}_{2}$. If $\mathrm{m}_{1}\left(g_{n}^{\prime}\right) \sim \mathrm{m}_{1}\left(w_{n}\right)$, then set $g_{n}^{\prime \prime}=\left\langle w_{n}, 1\right\rangle$. Otherwise choose positive integers $i_{n}$ such that

$$
i_{n} \sim\left[\mathrm{m}_{1}\left(g_{n}^{\prime}\right)-\mathrm{m}_{1}\left(w_{n}\right)\right] \frac{\mathrm{D}_{1}\left(w_{n}\right)}{\mathrm{t}_{1}(c)} .
$$

and take $g_{n}^{\prime \prime}=\left\langle w_{n}, c^{i_{n}}\right\rangle$. Either way, $\mathrm{m}_{1}\left(g_{n}^{\prime \prime}\right) \sim \mathrm{m}_{1}\left(g_{n}^{\prime}\right)$, which means that $g_{n}^{\prime \prime} x_{1} \rightarrow \zeta_{1}^{\prime}$. By hypothesis, $\partial X_{2}<\partial X_{1}$, so $g_{n}^{\prime \prime} x_{2} \rightarrow \zeta_{2}$, and $\lim _{n \rightarrow \infty} \mathrm{m}_{2}\left(g_{n}^{\prime \prime}\right)=\lim _{n \rightarrow \infty} \mathrm{m}_{2}\left(g_{n}\right)$. So

$$
\mathrm{m}_{1}\left(g_{n}^{\prime \prime}\right)-\mathrm{m}_{1}\left(g_{n}\right) \sim\left[\mathrm{m}_{2}\left(g_{n}^{\prime \prime}\right)-\mathrm{m}_{2}\left(g_{n}\right)\right] \frac{\mathrm{t}_{1}(c)}{\mathrm{t}_{2}(c)} \frac{\mathrm{D}_{2}\left(w_{n}\right)}{\mathrm{D}_{1}\left(w_{n}\right)} \rightarrow 0
$$


because Lemma 4.4 guarantees the ratio of the $D_{i}$ 's remains bounded. But this of course means that $\mathrm{m}_{1}\left(g_{n}\right) \rightarrow \zeta_{1}^{\prime}$ contradicting the fact that $\zeta_{1}^{\prime} \neq \zeta_{1}$.

\section{REFERENCES}

[1] M. Bestvina, Local homology properties of boundaries of groups, Michigan Math. J., 43 (1996), pp. 123-139.

[2] P. L. Bowers and K. Ruane, Boundaries of nonpositively curved groups of the form $G \times \mathbb{Z}^{n}$, Glasgow Math. J., 38 (1996), pp. 177-189.

[3] M. R. Bridson and A. Haffliger, Metric spaces of non-positive curvature, vol. 319 of Grundlehren der Mathematischen Wissenschaften [Fundamental Principles of Mathematical Sciences], Springer-Verlag, Berlin, 1999.

[4] T. A. Chapman, Lectures on Hilbert cube manifolds, American Mathematical Society, Providence, R. I., 1976. Expository lectures from the CBMS Regional Conference held at Guilford College, October 11-15, 1975, Regional Conference Series in Mathematics, No. 28.

[5] C. B. Croke And B. Kleiner, Spaces with nonpositive curvature and their ideal boundaries, Topology, 39 (2000), pp. $549-$ 556.

[6] The geodesic flow of a nonpositively curved graph manifold, Geom. Funct. Anal., 12 (2002), pp. 479-545.

[7] S. Ferry, Shape equivalence does not imply CE equivalence, Proc. Amer. Math. Soc., 80 (1980), pp. 154-156.

[8] R. Geoghegan, The shape of a group - connections between shape theory and the homology of groups, in Geometric and algebraic topology, vol. 18 of Banach Center Publ., PWN, Warsaw, 1986, pp. 271-280.

[9] R. Geoghegan and P. Ontaneda, Boundaries of cocompact proper CAT(0) spaces, Topology, 46 (2007), pp. 129-137.

[10] T. Hosaka, On equivariant homeomorphisms of boundaries of cat(0) groups. Preprint, 2010.

[11] G. C. Hruska And B. Kleiner, Hadamard spaces with isolated flats, Geom. Topol., 9 (2005), pp. 1501-1538 (electronic). With an appendix by the authors and Mohamad Hindawi.

[12] B. Kleiner And B. Leeb, Rigidity of quasi-isometries for symmetric spaces and euclidean buildings, Inst. Hautes tudes Sci. Publ. Math., (1997), pp. 115-197.

[13] G. Link, Asymptotic geometry in products of Hadamard spaces with rank one isometries, Geom. Topol., 14 (2010), pp. 1063-1094.

[14] J. Milnor, A note on curvature and fundamental group, J. Differential Geometry, 2 (1968), pp. 1-7.

[15] C. Mooney, Examples of non-rigid CAT(0) groups from the category of knot groups, Algebr. Geom. Topol., 8 (2008), pp. $1666-1689$.

[16] - All CAT(0) boundaries of a group of the form $H \times K$ are CE equivalent, Fund. Math., 203 (2009), pp. 97-106.

[17] — Generalizing the Croke-Kleiner construction, Topology Appl., 157 (2010), pp. 1168-1181.

[18] P. Ontaneda, Cocompact CAT(0) spaces are almost geodesically complete, Topology, 44 (2005), pp. 47-62.

[19] P. Papasoglu and E. Swenson, Boundaries and JSJ decompositions of CAT(0)-groups, Geom. Funct. Anal., 19 (2009), pp. 559-590.

[20] K. E. RuAne, The angle question, Topology Appl., 110 (2001), pp. 99-111. Geometric topology and geometric group theory (Milwaukee, WI, 1997).

[21] R. B. Sher, Realizing cell-like maps in Euclidean space, General Topology and Appl., 2 (1972), pp. 75-89.

[22] D. Staley, Erratic boundary images of CAT(0) geodesics under G-equivariant maps. Preprint, arXiv:0911.2442, 2009.

[23] A. S. Švarc, A volume invariant of coverings, Dokl. Akad. Nauk SSSR (N.S.), 105 (1955), pp. 32-34.

[24] E. L. Swenson, A cut point theorem for CAT(0) groups, J. Differential Geom., 53 (1999), pp. 327-358.

[25] J. M. WiLson, A CAT(0) group with uncountably many distinct boundaries, J. Group Theory, 8 (2005), pp. 229-238. 